Supplemental Information for

\title{
Determining the Ionization Constants of Organic Acids Using Fluorine Gauche Effect
}

\author{
Bright U. Emenike* and Simran S. Dhami \\ Department of Chemistry \& Physics \\ State University of New York, Old Westbury, New York \\ e-mail: emenikeb@oldwestbury.edu \\ phone: $516-628-5664$
}

\section{Table of Content}

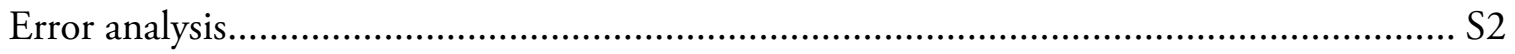

Table S1: Vicinal coupling constants as a function of concentration for compound 1 .... S2

Table S2. General formulas for propagation error calculations: ................................ S3

Graph of vicinal coupling constants as a function of concentration ................................ S3

Table S3. Computation and error analysis summary ............................................ S4

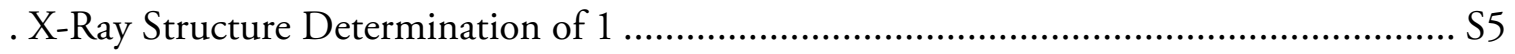

Figure S1. Crystal structure of compound 1. The ellipsoid contour of probability level is

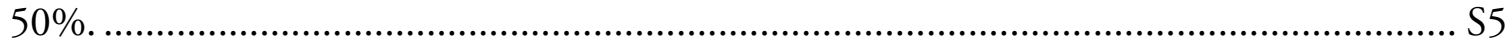

Table S4. Crystal data and structure refinement for compound 1 (code name: A13007)... S6

Table S5. Atomic coordinates ( $x 10^{4}$ ) and equivalent isotropic displacement parameters $\left(\AA^{2} \times 10^{3}\right)$ for $A 13007$. U(eq) is defined as one third of the trace of the orthogonalized

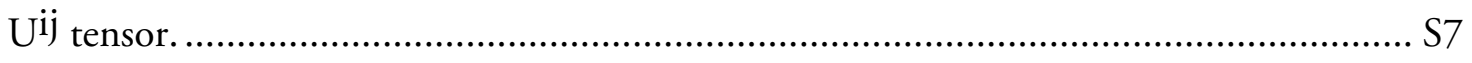

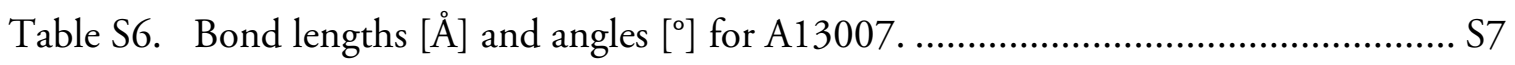

Table S7. Anisotropic displacement parameters (Å2x 103) for A13007. The anisotropic displacement factor exponent takes the form:

Table S8. Hydrogen coordinates ( $\mathrm{x}$ 104) and isotropic displacement parameters ( $\mathrm{\AA} 2 \mathrm{x}$

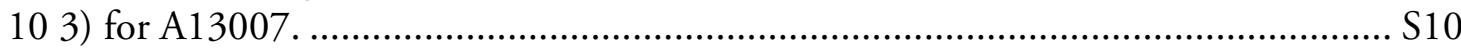

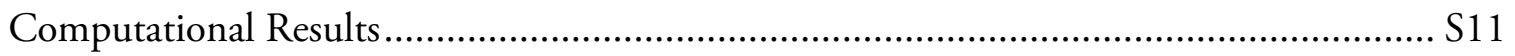


The xyz coordinates for the gauche conformer of 1 optimized at wB97xD/6-31+G(d)levels

The xyz coordinates for the trans conformer of 1 optimized at wB97xD/6-31+G(d)-levels

The xyz coordinates for the gauche conformer of 2 optimized at $w B 97 x D / 6-31+G(d)-$ levels

Table S10 Summary of computational Results

The xyz coordinates for the trans conformer of 2 optimized at wB97xD/6-31+G(d)-levels

References

Proton and Carbon NMR Spectrums

\section{Error analysis}

The origins of errors in the measurements of chemical shifts stem predominantly from the interactions between the components in solution. ${ }^{1,2}$ Errors due to solvation, concentration, and temperature are often the main culprits, leading to errors that are typically larger than the precision of the spectrometers. ${ }^{1}$ Such errors might exist in the acids ( 1 and 2) by virtue of their ability to form intermolecular hydrogen bonds. This is of less concern in the anions because one expects electrostatic repulsion and the absence of the hydrogen bonds to minimize intermolecular interactions. Hydrogen bonds are in general concentrationdependent. Given that intermolecular NH/O and intramolecular NH/F hydrogen bonds might influence the fluorine gauche effect, we decided to investigate the changes in the ${ }^{3} J_{\mathrm{HF}}$ value as a function concentration. The result is shown below.

\section{Table S1: Vicinal coupling constants as a function of concentration for compound 1}

\begin{tabular}{cc}
\hline Concentration $(\mathrm{mM})$ & $3_{\mathrm{HF}}(\mathrm{Hz})$ \\
\hline 45 & 26.76 \\
89 & 26.84 \\
115 & 26.82 \\
154 & 26.76 \\
230 & 26.76 \\
400 & 26.76 \\
\hline
\end{tabular}

As can be seen in the table, the changes in the vicinal coupling constants as a function of concentration is negligible, indicating that intermolecular hydrogen bond seems to have a 
negligible on the gauche effects of 1 . The average ${ }^{3} J_{\mathrm{HF}}$ value is $26.78 \mathrm{~Hz}$ across all concentration, with a standard deviation of $0.04 \mathrm{~Hz}$. Conservatively, we used $26.8 \mathrm{~Hz}$ as the $3 J_{\mathrm{HF}}$ value for compound 1 with an error of $0.1 \mathrm{~Hz}$. This error was applied to all measured vicinal coupling constants.

Table S2. General formulas for propagation error calculations:

\begin{tabular}{|c|c|c|}
\hline Process & Value & Uncertainty \\
\hline Average & $\bar{x}=\frac{x_{1}+x_{2}+x_{3}+\ldots+x_{N V}}{N}$ & $\sigma_{x}=\sqrt{\frac{\left(x_{1}-\bar{x}\right)^{2}+\left(x_{2}-\bar{x}\right)^{2}+\ldots+\left(x_{N}-\bar{x}\right)^{2}}{N-1}}$ \\
\hline Addition & $\bar{z}=\bar{x}+\bar{y}$ & $\sigma_{z}=\sqrt{\left(\sigma_{x}\right)^{2}+\left(\sigma_{y}\right)^{2}}$ \\
\hline Subtraction & $\bar{z}=\bar{x}-\bar{y}$ & $\sigma_{z}=\sqrt{\left(\sigma_{x}\right)^{2}+\left(\sigma_{y}\right)^{2}}$ \\
\hline Multiplication & $\bar{z}=\bar{x} * \bar{y}$ & $\sigma_{z}=\bar{z} * \sqrt{\left(\frac{\sigma_{x}}{\bar{x}}\right)^{2}+\left(\frac{\sigma_{y}}{\bar{y}}\right)^{2}}$ \\
\hline Division & $\bar{z}=\bar{x} \div \bar{y}$ & $\sigma_{z}=\bar{z} * \sqrt{\left(\frac{\sigma_{x}}{\bar{x}}\right)^{2}+\left(\frac{\sigma_{y}}{\bar{y}}\right)^{2}}$ \\
\hline
\end{tabular}

Graph of vicinal coupling constants as a function of concentration

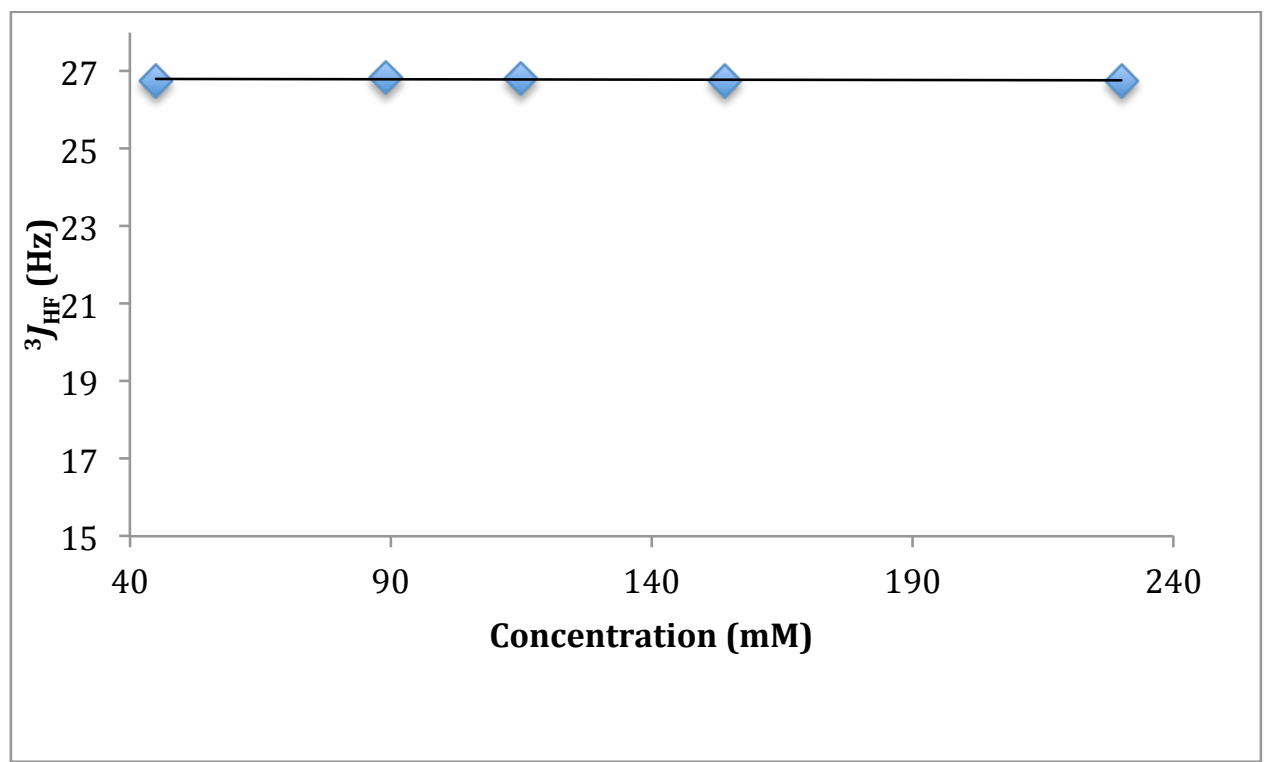


Table S3. Computation and error analysis summary

\begin{tabular}{|c|c|c|c|c|c|c|c|c|c|c|c|c|c|c|c|}
\hline \multirow{2}{*}{$\begin{array}{l}\text { st } \\
\text { d }\end{array}$} & \multirow[b]{2}{*}{ Test } & \multicolumn{3}{|c|}{$(\mathrm{Hz})$} & \multicolumn{2}{|c|}{$(\mathrm{Hz})$} & \multicolumn{2}{|c|}{$(\mathrm{Hz})$} & \multirow[b]{2}{*}{$\left(K_{\mathrm{rel}}\right)$} & \multirow[b]{2}{*}{ Error } & \multirow[b]{2}{*}{$K_{\mathrm{a}}$} & \multirow[b]{2}{*}{$K_{\mathrm{b}}$} & \multirow[b]{2}{*}{ Error $\left(K_{\mathrm{b}}\right)$} & \multirow[b]{2}{*}{$\mathrm{p} K_{\mathrm{b}}$} & \multirow[b]{2}{*}{ Error } \\
\hline & & ${ }^{3} J_{\mathrm{AH}}$ & ${ }^{3} J_{\mathrm{A}}$ & ${ }^{3} J_{\text {obs }}$ & ${ }^{3} J_{\text {obs }}$ & Error & ${ }^{3} J_{\mathrm{A}}$ & Error & & & & & & & \\
\hline \multirow[t]{9}{*}{1} & Succinimide & 26.8 & 18.4 & 24.8 & 2.0 & 0.14 & 6.4 & 0.14 & 0.098 & 0.010 & $6.30957 \mathrm{E}-17$ & $6.461 \mathrm{E}-16$ & $6.7011 \mathrm{E}-17$ & 15.2 & 0.05 \\
\hline & Diethyl malonate & 26.8 & 18.4 & 21.6 & 5.2 & 0.14 & 3.2 & 0.14 & 2.641 & 0.192 & $6.30957 \mathrm{E}-17$ & $2.38942 \mathrm{E}-17$ & $1.73588 \mathrm{E}-18$ & 16.6 & 0.03 \\
\hline & 2-Naphthol & 26.8 & 18.4 & 20.4 & 6.4 & 0.14 & 2.0 & 0.14 & 10.240 & 1.062 & $6.30957 \mathrm{E}-17$ & $6.16169 \mathrm{E}-18$ & $6.39067 \mathrm{E}-19$ & 17.2 & 0.05 \\
\hline & Diphenylacetone & 26.8 & 18.4 & 18.8 & 8.0 & 0.14 & 0.4 & 0.14 & 400.000 & 198.237 & $6.30957 \mathrm{E}-17$ & $1.57739 \mathrm{E}-19$ & $7.81745 \mathrm{E}-20$ & 18.8 & 0.22 \\
\hline & Benzimidazole & 26.8 & 18.4 & 21.2 & 5.6 & 0.14 & 2.8 & 0.14 & 4.000 & 0.316 & $6.30957 \mathrm{E}-17$ & $1.57739 \mathrm{E}-17$ & $1.24704 \mathrm{E}-18$ & 16.8 & 0.03 \\
\hline & estriol & 26.8 & 18.4 & 19.0 & 7.8 & 0.14 & 0.6 & 0.14 & 169.000 & 55.932 & $6.30957 \mathrm{E}-17$ & $3.73348 \mathrm{E}-19$ & $1.23562 \mathrm{E}-19$ & 18.4 & 0.14 \\
\hline & thioacetamide & 26.8 & 18.4 & 19.2 & 7.6 & 0.14 & 0.8 & 0.14 & 90.250 & 22.459 & $6.30957 \mathrm{E}-17$ & $6.99122 \mathrm{E}-19$ & $1.7398 \mathrm{E}-19$ & 18.2 & 0.11 \\
\hline & 2-methoxyphenol & 26.8 & 18.4 & 19.6 & 7.2 & 0.14 & 1.2 & 0.14 & 36.000 & 6.022 & $6.30957 \mathrm{E}-17$ & $1.75266 \mathrm{E}-18$ & $2.93163 \mathrm{E}-19$ & 17.8 & 0.07 \\
\hline & $\begin{array}{c}\text { acid } \\
\text { 4-chloro-3- }\end{array}$ & 27.6 & 21.6 & 26.0 & 1.6 & 0.14 & 4.4 & 0.14 & 0.132 & 0.017 & 5.01187E-09 & 3.79023E-08 & 4.99064E-09 & 7.4 & 0.06 \\
\hline \multirow[t]{4}{*}{2} & $\begin{array}{l}\text { nitrobenzoic acid } \\
\text { 3-chlorobenzoic }\end{array}$ & 27.6 & 21.6 & 24.0 & 3.6 & 0.14 & 2.4 & 0.14 & 2.250 & 0.223 & 5.01187E-09 & $2.2275 \mathrm{E}-09$ & $2.20851 \mathrm{E}-10$ & 8.7 & 0.04 \\
\hline & $\begin{array}{l}\text { acid } \\
\text { 4-chlorobenzoic }\end{array}$ & 27.6 & 21.6 & 22.8 & 4.8 & 0.14 & 1.2 & 0.14 & 16.000 & 2.721 & $5.01187 \mathrm{E}-09$ & $3.13242 \mathrm{E}-10$ & $5.32729 \mathrm{E}-11$ & 9.5 & 0.07 \\
\hline & acid & 27.6 & 21.6 & 22.4 & 5.2 & 0.14 & 0.8 & 0.14 & 42.250 & 10.579 & $5.01187 \mathrm{E}-09$ & $1.18624 \mathrm{E}-10$ & $2.97034 \mathrm{E}-11$ & 9.9 & 0.11 \\
\hline & 4-Nitrophenol & 27.6 & 21.6 & 22.0 & 5.6 & 0.14 & 0.4 & 0.14 & 196.000 & 97.262 & $5.01187 \mathrm{E}-09$ & $2.55708 \mathrm{E}-11$ & $1.26891 \mathrm{E}-11$ & 10.6 & 0.22 \\
\hline
\end{tabular}




\section{. X-Ray Structure Determination of 1}

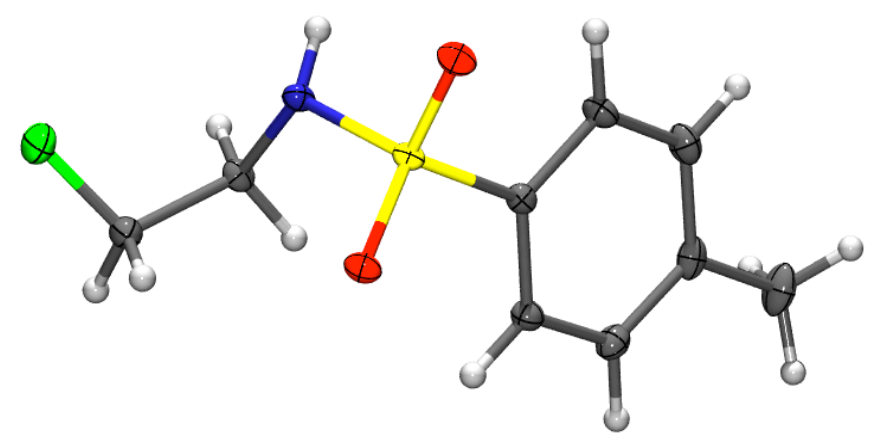

\section{Figure S1. Crystal structure of compound 1. The ellipsoid contour of probability level is $50 \%$. Crystal Preparation:}

The crystals of compound 1 were grown by the Vapor Diffusion method. A solution of -30 $\mathrm{mg}$ of compound 1 (in dichloromethane) in an open one-dram vial was placed in a slightly larger vial containing hexane, which was subsequently sealed. After a few days, crystals (suitable for $\mathrm{x}$-ray crystallography) developed in the one-dram vial, which was analyzed for quality.

\section{X-ray Data Collection:}

Low-temperature diffraction data (-and -scans) were collected on a Bruker Kappa four-circle diffractometer coupled to a Bruker APEX II CCD detector with graphite-monochromated Mo K radiation $(=0.71073 \AA$ ) for the structure of compound A13007. The structure was solved by direct methods using SHELXS and refined against $F^{2}$ on all data by full-matrix least squares with SHELXL-2013 using established refinement techniques. All non-hydrogen atoms were refined anisotropically. Unless otherwise noted, all hydrogen atoms were included into the model at geometrically calculated positions and refined using a riding model. The isotropic displacement parameters of all hydrogen atoms were fixed to 1.2 times the $U$ value of the atoms they are linked to (1.5 times for methyl groups). All disordered atoms were refined with the help of similarity restraints as well as rigid bond restraints on the anisotropic displacement parameters.

Compound A13007 crystallizes in the monoclinic space group $P 2_{1} / n$ with one molecule in the asymmetric unit. The coordinates for the hydrogen atom bound to N1 was taken from the difference Fourier synthesis and the hydrogen atom were subsequently refined semi-freely with the help of a distance restraint. 
Table S4. Crystal data and structure refinement for compound 1 (code name: A13007).

\begin{tabular}{|c|c|c|}
\hline Identification code & A13007 & \\
\hline Empirical formula & C9 $\mathrm{H} 12 \mathrm{~F} \mathrm{~N} \mathrm{O} 2 \mathrm{~S}$ & \\
\hline Formula weight & 217.26 & \\
\hline Temperature & $100(2) \mathrm{K}$ & \\
\hline Wavelength & $0.71073 \AA$ & \\
\hline Crystal system & Monoclinic & \\
\hline Space group & $\mathrm{P} 21 / \mathrm{n}$ & \\
\hline Unit cell dimensions & $a=5.8237(3) \AA$ & á $=90^{\circ}$. \\
\hline & $\mathrm{b}=8.8525(4) \AA$ & $\hat{\mathrm{a}}=92.943(3)^{\circ}$. \\
\hline & $\mathrm{c}=19.1618(10) \AA$ & $\tilde{\mathrm{a}}=90^{\circ}$. \\
\hline Volume & $986.57(9) \AA 3$ & \\
\hline $\mathrm{Z}$ & 4 & \\
\hline Density (calculated) & $1.463 \mathrm{Mg} / \mathrm{m} 3$ & \\
\hline Absorption coefficient & $0.316 \mathrm{~mm}-1$ & \\
\hline $\mathrm{F}(000)$ & 456 & \\
\hline Crystal size & $0.500 \times 0.400 \times 0.200 \mathrm{~mm} 3$ & \\
\hline Theta range for data collection & 2.535 to $30.554^{\circ}$. & \\
\hline Index ranges & $\begin{array}{l}-8<=\mathrm{h}<=8, \quad-12<=\mathrm{k}<=12, \quad- \\
27<=\mathrm{l}<=27\end{array}$ & \\
\hline Reflections collected & 45362 & \\
\hline Independent reflections & $3019[\mathrm{R}($ int $)=0.0318]$ & \\
\hline $\begin{array}{l}\text { Completeness to theta }= \\
25.242^{\circ}\end{array}$ & $99.90 \%$ & \\
\hline Absorption correction & Semi-empirical from equivalents & \\
\hline Max. and min. transmission & 0.7461 and 0.6846 & \\
\hline Refinement method & Full-matrix least-squares on F2 & \\
\hline Data / restraints / parameters & $3019 / 1 / 131$ & \\
\hline Goodness-of-fit on F2 & 1.049 & \\
\hline Final R indices $[\mathrm{I}>2 \operatorname{sigma}(\mathrm{I})]$ & $\mathrm{R} 1=0.0283, \mathrm{wR} 2=0.0808$ & \\
\hline $\mathrm{R}$ indices (all data) & $\mathrm{R} 1=0.0292, \mathrm{wR} 2=0.0817$ & \\
\hline Extinction coefficient & $\mathrm{n} / \mathrm{a}$ & \\
\hline Largest diff. peak and hole & 0.471 and -0.382 e. $\AA-3$ & \\
\hline
\end{tabular}


Table S5. Atomic coordinates ( $\times 10^{4}$ ) and equivalent isotropic displacement parameters $\left(\AA^{2} \times 10^{3}\right)$ for $A 13007$. U(eq) is defined as one third of the trace of the orthogonalized $U^{i j}$ tensor.

\begin{tabular}{lrrrr}
\hline- & & & & \\
& & $\mathrm{x}$ & $\mathrm{U}(\mathrm{eq})$ \\
\hline $\mathrm{S}(1)$ & & & & \\
$\mathrm{O}(1)$ & $11773(1)$ & $4451(1)$ & $2123(1)$ & $10(1)$ \\
$\mathrm{O}(2)$ & $14237(1)$ & $4317(1)$ & $2112(1)$ & $16(1)$ \\
$\mathrm{C}(1)$ & $10413(1)$ & $3088(1)$ & $2140(1)$ & $14(1)$ \\
$\mathrm{C}(2)$ & $10795(2)$ & $5496(1)$ & $1385(1)$ & $12(1)$ \\
$\mathrm{C}(3)$ & $8626(2)$ & $5222(1)$ & $1068(1)$ & $14(1)$ \\
$\mathrm{C}(4)$ & $7903(2)$ & $6083(1)$ & $491(1)$ & $17(1)$ \\
$\mathrm{C}(5)$ & $9311(2)$ & $7197(1)$ & $223(1)$ & $17(1)$ \\
$\mathrm{C}(6)$ & $11469(2)$ & $7452(1)$ & $553(1)$ & $18(1)$ \\
$\mathrm{C}(7)$ & $12219(2)$ & $6618(1)$ & $1134(1)$ & $16(1)$ \\
$\mathrm{N}(1)$ & $8498(2)$ & $8104(1)$ & $-405(1)$ & $25(1)$ \\
$\mathrm{C}(8)$ & $11250(1)$ & $5420(1)$ & $2814(1)$ & $12(1)$ \\
$\mathrm{C}(9)$ & $8821(2)$ & $5772(1)$ & $2944(1)$ & $13(1)$ \\
$\mathrm{F}(1)$ & $7791(2)$ & $4653(1)$ & $3429(1)$ & $17(1)$ \\
& $8875(1)$ & $4783(1)$ & $4095(1)$ & $30(1)$ \\
\hline
\end{tabular}

Table S6. Bond lengths $[\AA ̊]$ and angles $\left[^{\circ}\right]$ for A13007.

\begin{tabular}{ll}
\hline $\mathrm{S}(1)-\mathrm{O}(1)$ & $1.4410(7)$ \\
$\mathrm{S}(1)-\mathrm{O}(2)$ & $1.4446(7)$ \\
$\mathrm{S}(1)-\mathrm{N}(1)$ & $1.6195(9)$ \\
$\mathrm{S}(1)-\mathrm{C}(1)$ & $1.7594(10)$ \\
$\mathrm{C}(1)-\mathrm{C}(2)$ & $1.3942(13)$ \\
$\mathrm{C}(1)-\mathrm{C}(6)$ & $1.3963(13)$ \\
$\mathrm{C}(2)-\mathrm{C}(3)$ & $1.3897(14)$ \\
$\mathrm{C}(2)-\mathrm{H}(2)$ & 0.9500 \\
$\mathrm{C}(3)-\mathrm{C}(4)$ & $1.3974(15)$ \\
$\mathrm{C}(3)-\mathrm{H}(3)$ & 0.9500 \\
$\mathrm{C}(4)-\mathrm{C}(5)$ & $1.3960(15)$ \\
$\mathrm{C}(4)-\mathrm{C}(7)$ & $1.5030(15)$ \\
$\mathrm{C}(5)-\mathrm{C}(6)$ & $1.3870(14)$ \\
$\mathrm{C}(5)-\mathrm{H}(5)$ & 0.9500 \\
$\mathrm{C}(6)-\mathrm{H}(6)$ & 0.9500 \\
$\mathrm{C}(7)-\mathrm{H}(7 \mathrm{~A})$ & 0.9800
\end{tabular}




\begin{tabular}{|c|c|}
\hline $\mathrm{C}(7)-\mathrm{H}(7 \mathrm{~B})$ & 0.9800 \\
\hline $\mathrm{C}(7)-\mathrm{H}(7 \mathrm{C})$ & 0.9800 \\
\hline $\mathrm{N}(1)-\mathrm{C}(8)$ & $1.4820(12)$ \\
\hline $\mathrm{N}(1)-\mathrm{H}(1 \mathrm{~N})$ & $0.846(12)$ \\
\hline $\mathrm{C}(8)-\mathrm{C}(9)$ & $1.5046(14)$ \\
\hline $\mathrm{C}(8)-\mathrm{H}(8 \mathrm{~A})$ & 0.9900 \\
\hline $\mathrm{C}(8)-\mathrm{H}(8 \mathrm{~B})$ & 0.9900 \\
\hline $\mathrm{C}(9)-\mathrm{F}(1)$ & $1.3990(12)$ \\
\hline $\mathrm{C}(9)-\mathrm{H}(9 \mathrm{~A})$ & 0.9900 \\
\hline $\mathrm{C}(9)-\mathrm{H}(9 \mathrm{~B})$ & 0.9900 \\
\hline $\mathrm{O}(1)-\mathrm{S}(1)-\mathrm{O}(2)$ & $118.63(4)$ \\
\hline $\mathrm{O}(1)-\mathrm{S}(1)-\mathrm{N}(1)$ & $106.55(5)$ \\
\hline $\mathrm{O}(2)-\mathrm{S}(1)-\mathrm{N}(1)$ & $107.31(4)$ \\
\hline $\mathrm{O}(1)-\mathrm{S}(1)-\mathrm{C}(1)$ & $108.25(4)$ \\
\hline $\mathrm{O}(2)-\mathrm{S}(1)-\mathrm{C}(1)$ & $107.57(4)$ \\
\hline $\mathrm{N}(1)-S(1)-\mathrm{C}(1)$ & $108.16(4)$ \\
\hline $\mathrm{C}(2)-\mathrm{C}(1)-\mathrm{C}(6)$ & $120.94(9)$ \\
\hline$C(2)-C(1)-S(1)$ & $120.50(7)$ \\
\hline$C(6)-C(1)-S(1)$ & $118.55(7)$ \\
\hline $\mathrm{C}(3)-\mathrm{C}(2)-\mathrm{C}(1)$ & $118.82(9)$ \\
\hline $\mathrm{C}(3)-\mathrm{C}(2)-\mathrm{H}(2)$ & 120.6 \\
\hline $\mathrm{C}(1)-\mathrm{C}(2)-\mathrm{H}(2)$ & 120.6 \\
\hline$C(2)-C(3)-C(4)$ & $121.32(9)$ \\
\hline $\mathrm{C}(2)-\mathrm{C}(3)-\mathrm{H}(3)$ & 119.3 \\
\hline $\mathrm{C}(4)-\mathrm{C}(3)-\mathrm{H}(3)$ & 119.3 \\
\hline$C(5)-C(4)-C(3)$ & $118.65(9)$ \\
\hline$C(5)-C(4)-C(7)$ & $121.03(10)$ \\
\hline$C(3)-C(4)-C(7)$ & $120.32(10)$ \\
\hline$C(6)-C(5)-C(4)$ & $121.08(9)$ \\
\hline $\mathrm{C}(6)-\mathrm{C}(5)-\mathrm{H}(5)$ & 119.5 \\
\hline $\mathrm{C}(4)-\mathrm{C}(5)-\mathrm{H}(5)$ & 119.5 \\
\hline$C(5)-C(6)-C(1)$ & $119.18(9)$ \\
\hline $\mathrm{C}(5)-\mathrm{C}(6)-\mathrm{H}(6)$ & 120.4 \\
\hline $\mathrm{C}(1)-\mathrm{C}(6)-\mathrm{H}(6)$ & 120.4 \\
\hline $\mathrm{C}(4)-\mathrm{C}(7)-\mathrm{H}(7 \mathrm{~A})$ & 109.5 \\
\hline $\mathrm{C}(4)-\mathrm{C}(7)-\mathrm{H}(7 \mathrm{~B})$ & 109.5 \\
\hline $\mathrm{H}(7 \mathrm{~A})-\mathrm{C}(7)-\mathrm{H}(7 \mathrm{~B})$ & 109.5 \\
\hline $\mathrm{C}(4)-\mathrm{C}(7)-\mathrm{H}(7 \mathrm{C})$ & 109.5 \\
\hline $\mathrm{H}(7 \mathrm{~A})-\mathrm{C}(7)-\mathrm{H}(7 \mathrm{C})$ & 109.5 \\
\hline $\mathrm{H}(7 \mathrm{~B})-\mathrm{C}(7)-\mathrm{H}(7 \mathrm{C})$ & 109.5 \\
\hline $\mathrm{C}(8)-\mathrm{N}(1)-\mathrm{S}(1)$ & $118.03(7)$ \\
\hline $\mathrm{C}(8)-\mathrm{N}(1)-\mathrm{H}(1 \mathrm{~N})$ & $113.9(10)$ \\
\hline$S(1)-N(1)-H(1 N)$ & $111.1(10)$ \\
\hline
\end{tabular}




$\begin{array}{ll}\mathrm{N}(1)-\mathrm{C}(8)-\mathrm{C}(9) & 112.31(8) \\ \mathrm{N}(1)-\mathrm{C}(8)-\mathrm{H}(8 \mathrm{~A}) & 109.1 \\ \mathrm{C}(9)-\mathrm{C}(8)-\mathrm{H}(8 \mathrm{~A}) & 109.1 \\ \mathrm{~N}(1)-\mathrm{C}(8)-\mathrm{H}(8 \mathrm{~B}) & 109.1 \\ \mathrm{C}(9)-\mathrm{C}(8)-\mathrm{H}(8 \mathrm{~B}) & 109.1 \\ \mathrm{H}(8 \mathrm{~A})-\mathrm{C}(8)-\mathrm{H}(8 \mathrm{~B}) & 107.9 \\ \mathrm{~F}(1)-\mathrm{C}(9)-\mathrm{C}(8) & 109.52(8) \\ \mathrm{F}(1)-\mathrm{C}(9)-\mathrm{H}(9 \mathrm{~A}) & 109.8 \\ \mathrm{C}(8)-\mathrm{C}(9)-\mathrm{H}(9 \mathrm{~A}) & 109.8 \\ \mathrm{~F}(1)-\mathrm{C}(9)-\mathrm{H}(9 \mathrm{~B}) & 109.8 \\ \mathrm{C}(8)-\mathrm{C}(9)-\mathrm{H}(9 \mathrm{~B}) & 109.8 \\ \mathrm{H}(9 \mathrm{~A})-\mathrm{C}(9)-\mathrm{H}(9 \mathrm{~B}) & 108.2\end{array}$

Symmetry transformations used to generate equivalent atoms:

Table S7. Anisotropic displacement parameters (Å2x 103) for A13007. The anisotropic displacement factor exponent takes the form:

\begin{tabular}{|c|c|c|c|c|c|c|}
\hline & $\mathrm{U}^{11}$ & $\mathrm{U}^{22}$ & U33 & $\mathrm{U}^{23}$ & $\mathrm{U}^{13}$ & $\mathrm{U}^{12}$ \\
\hline$S(1)$ & $9(1)$ & $9(1)$ & $14(1)$ & $0(1)$ & $0(1)$ & $0(1)$ \\
\hline $\mathrm{O}(1)$ & $9(1)$ & $17(1)$ & $21(1)$ & $0(1)$ & $1(1)$ & $2(1)$ \\
\hline $\mathrm{O}(2)$ & $14(1)$ & $9(1)$ & $18(1)$ & $0(1)$ & $1(1)$ & $-2(1)$ \\
\hline $\mathrm{C}(1)$ & $11(1)$ & $10(1)$ & $13(1)$ & $0(1)$ & $1(1)$ & $1(1)$ \\
\hline $\mathrm{C}(2)$ & $12(1)$ & $15(1)$ & $15(1)$ & $0(1)$ & $0(1)$ & $-1(1)$ \\
\hline$C(3)$ & $16(1)$ & $19(1)$ & $15(1)$ & $-1(1)$ & $-1(1)$ & $3(1)$ \\
\hline C(4) & $22(1)$ & $15(1)$ & $13(1)$ & $1(1)$ & $4(1)$ & $7(1)$ \\
\hline$C(5)$ & $21(1)$ & $14(1)$ & $20(1)$ & $4(1)$ & $5(1)$ & $0(1)$ \\
\hline$C(6)$ & $14(1)$ & $14(1)$ & $19(1)$ & $2(1)$ & $2(1)$ & $-2(1)$ \\
\hline $\mathrm{C}(7)$ & $37(1)$ & $22(1)$ & $15(1)$ & $5(1)$ & $2(1)$ & $10(1)$ \\
\hline $\mathrm{N}(1)$ & $11(1)$ & $11(1)$ & $14(1)$ & $-2(1)$ & 1(1) & $-1(1)$ \\
\hline C(8) & $12(1)$ & $13(1)$ & $16(1)$ & $0(1)$ & $2(1)$ & $2(1)$ \\
\hline C(9) & $14(1)$ & $21(1)$ & $15(1)$ & $1(1)$ & $1(1)$ & $-3(1)$ \\
\hline $\mathrm{F}(1)$ & $26(1)$ & $48(1)$ & $15(1)$ & $7(1)$ & $-3(1)$ & $-10(1)$ \\
\hline
\end{tabular}


Table S8. Hydrogen coordinates ( x 104) and isotropic displacement parameters $(\AA 2 \mathrm{x} 10$ 3) for A13007.

\begin{tabular}{|c|c|c|c|c|}
\hline - & $\mathrm{x}$ & $\mathrm{y}$ & $\mathrm{z}$ & $\mathrm{U}(\mathrm{eq})$ \\
\hline \multicolumn{5}{|l|}{ - } \\
\hline $\mathrm{H}(2)$ & 7660 & 4462 & 1243 & 17 \\
\hline $\mathrm{H}(3)$ & 6422 & 5909 & 275 & 20 \\
\hline $\mathrm{H}(5)$ & 12440 & 8210 & 377 & 22 \\
\hline $\mathrm{H}(6)$ & 13684 & 6809 & 1357 & 19 \\
\hline $\mathrm{H}(7 \mathrm{~A})$ & 7849 & 9062 & -252 & 37 \\
\hline $\mathrm{H}(7 \mathrm{~B})$ & 7317 & 7534 & -676 & 37 \\
\hline $\mathrm{H}(7 \mathrm{C})$ & 9797 & 8306 & -697 & 37 \\
\hline $\mathrm{H}(1 \mathrm{~N})$ & $12140(20)$ & $6170(15)$ & $2859(7)$ & 14 \\
\hline $\mathrm{H}(8 \mathrm{~A})$ & 7909 & 5775 & 2493 & 16 \\
\hline $\mathrm{H}(8 \mathrm{~B})$ & 8734 & 6796 & 3148 & 16 \\
\hline $\mathrm{H}(9 \mathrm{~A})$ & 6124 & 4849 & 3455 & 20 \\
\hline $\mathrm{H}(9 \mathrm{~B})$ & 7989 & 3615 & 3249 & 20 \\
\hline \multicolumn{5}{|c|}{ _ Table S9. Hydrogen bonds for A13007 [A and $\left.{ }^{\circ}\right]$. } \\
\hline D-H...A & $\mathrm{d}(\mathrm{D}-\mathrm{H})$ & $\mathrm{d}(\mathrm{H} \ldots \mathrm{A})$ & d(D...A) & $<(\mathrm{DHA})$ \\
\hline $\mathrm{N}(1)-\mathrm{H}(1 \mathrm{~N}) \ldots \mathrm{S}(1) \# 1$ & $0.846(12)$ & $2.972(13)$ & $3.7499(9)$ & $153.9(12)$ \\
\hline $\mathrm{N}(1)-\mathrm{H}(1 \mathrm{~N}) \ldots \mathrm{O}(2) \# 1$ & $0.846(12)$ & $2.215(12)$ & $3.0571(11)$ & $173.8(13)$ \\
\hline $\mathrm{C}(8)-\mathrm{H}(8 \mathrm{~A}) \ldots \mathrm{O}(1) \# 2$ & 0.99 & 2.57 & $3.2999(13)$ & 130.3 \\
\hline $\mathrm{C}(8)-\mathrm{H}(8 \mathrm{~B}) \ldots \mathrm{O}(1) \# 1$ & 0.99 & 2.59 & $3.3396(13)$ & 132.9 \\
\hline $\mathrm{C}(9)-\mathrm{H}(9 \mathrm{~B}) \ldots \mathrm{O}(2)$ & 0.99 & 2.65 & $3.2779(13)$ & 121.2 \\
\hline
\end{tabular}

Symmetry transformations used to generate equivalent atoms:

$\# 1-x+5 / 2, y+1 / 2,-z+1 / 2 \quad \# 2 x-1, y, z$ 


\section{Computational Results}

The xyz coordinates for the gauche conformer of 1 optimized at w $\mathrm{B} 97 \mathrm{xD} / 6$ $31+\mathrm{G}(\mathrm{d})$-levels

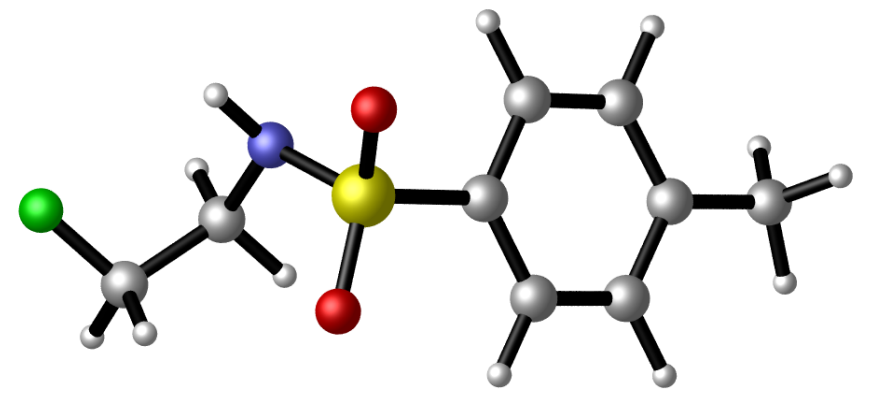

$\begin{array}{lccr}\text { S-S_3+4 } & -0.88377800 & 1.00375600 & 0.25569500 \\ \text { O-O_2 } & -0.98600200 & 2.35372100 & -0.27678200 \\ \text { O-O_2 } & -1.32846000 & 0.70197600 & 1.61304700 \\ \text { N-N_3 } & -1.66714500 & 0.04210700 & -0.86267200 \\ \text { H-H_ } & -2.40333900 & 0.57536900 & -1.31856800 \\ \text { C-C_3 } & -2.04269600 & -1.31706500 & -0.48395200 \\ \text { H-H_ } & -2.10778600 & -1.90533700 & -1.40506500 \\ \text { H-H_ } & -1.23738200 & -1.74768400 & 0.12083400 \\ \text { C-C_3 } & -3.35278700 & -1.41149900 & 0.27448300 \\ \text { H-H_- } & -3.58463900 & -2.45216200 & 0.52199600 \\ \text { H-H_- } & -3.34490700 & -0.79560500 & 1.17635900 \\ \text { F-F_ } & -4.37882800 & -0.93822500 & -0.55221500 \\ \text { C-C_R } & 0.79245100 & 0.41874100 & 0.11945000 \\ \text { C-C_R } & 1.39886900 & -0.18941200 & 1.21124900 \\ \text { C-C_R } & 1.47956500 & 0.59446600 & -1.08114200 \\ \text { C-C_R } & 2.71457800 & -0.63458400 & 1.09377100 \\ \text { H-H_ } & 0.84803700 & -0.30533400 & 2.13910900 \\ \text { C-C_R } & 2.78805900 & 0.14499100 & -1.18071600 \\ \text { H-H_ } & 0.99113300 & 1.07397000 & -1.92364200 \\ \text { C-C_R } & 3.42570600 & -0.47468800 & -0.09648700 \\ \text { H-H_ } & 3.19203300 & -1.11064100 & 1.94629700 \\ \text { H-H_ } & 3.32739400 & 0.27704800 & -2.11543700 \\ \text { C-C_3 } & 4.85602000 & -0.93447900 & -0.21696500 \\ \text { H-H_- } & 5.54159100 & -0.08029000 & -0.16782000 \\ \text { H-H_- } & 5.12499900 & -1.62478800 & 0.58773100 \\ \text { H-H_ } & 5.02989400 & -1.43977500 & -1.17254300\end{array}$


The xyz coordinates for the trans conformer of 1 optimized at w $\mathrm{B} 97 \mathrm{xD} / 6$ $31+\mathrm{G}(\mathrm{d})$-levels

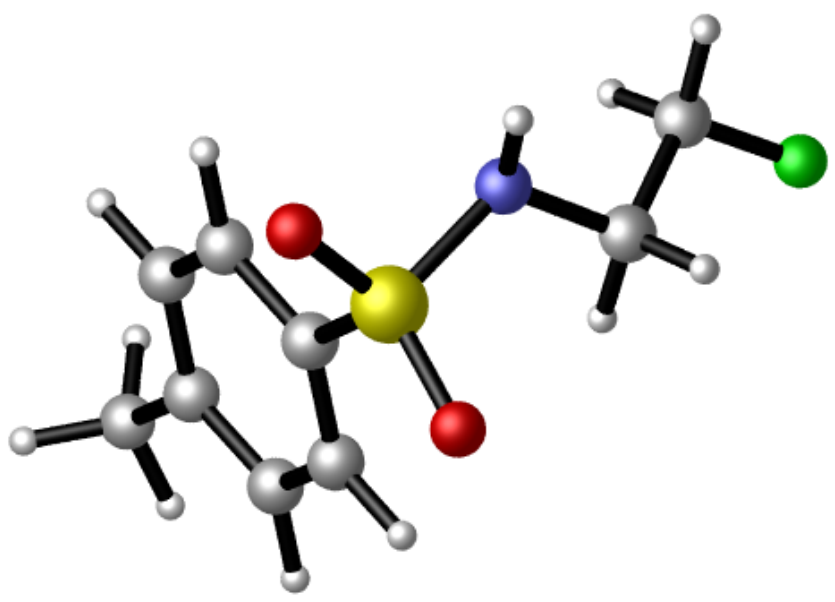

$\begin{array}{lrrr}\text { S-S_3+4 } & -0.88239400 & -1.49548100 & 0.07022100 \\ \text { O-O_2 } & -1.22303800 & -1.74501000 & 1.46470700 \\ \text { O-O_2 } & -0.87981100 & -2.57790400 & -0.90248400 \\ \text { N-N_3 } & -1.90160500 & -0.33879600 & -0.59406900 \\ \text { H-H_ } & -2.64179500 & -0.82434300 & -1.09570000 \\ \text { C-C_R } & 0.68064200 & -0.64786300 & 0.04386300 \\ \text { C-C_R } & 1.32291000 & -0.44493600 & -1.17721700 \\ \text { C-C_R } & 1.23749300 & -0.19663800 & 1.23407100 \\ \text { C-C_R } & 2.53722400 & 0.22517600 & -1.19467600 \\ \text { H-H_ } & 0.87579100 & -0.81359900 & -2.09502400 \\ \text { C-C_R } & 2.45744800 & 0.47610500 & 1.19696300 \\ \text { H-H_ } & 0.72678200 & -0.37954000 & 2.17411900 \\ \text { C-C_R } & 3.12253300 & 0.69542700 & -0.01045300 \\ \text { H-H_ } & 3.04496500 & 0.38447500 & -2.14277500 \\ \text { H-H_ } & 2.89691500 & 0.83120600 & 2.12533100 \\ \text { C-C_3 } & 4.45372700 & 1.40101600 & -0.05072400 \\ \text { H-H_ } & 5.25959700 & 0.69029400 & -0.26773800 \\ \text { H-H_ } & 4.47166300 & 2.16687000 & -0.83294000 \\ \text { H-H_ } & 4.68044500 & 1.88356300 & 0.90417300 \\ \text { C-C_3 } & -2.36364700 & 0.73843500 & 0.27967700 \\ \text { H-H_ } & -3.20232300 & 0.43377900 & 0.91627500 \\ \text { H-H_ } & -1.53992500 & 1.03378000 & 0.93613900 \\ \text { C-C_3 } & -2.74958600 & 1.92278600 & -0.59237400 \\ \text { H-H_ } & -1.88246200 & 2.30237900 & -1.13984000 \\ \text { F-F_ } & -3.23181000 & 2.94388700 & 0.22010600 \\ \text { H-H_ } & -3.54350800 & 1.66167800 & -1.30059200\end{array}$


The xyz coordinates for the gauche conformer of 2 optimized at wB97xD/6$31+\mathrm{G}(\mathrm{d})$-levels

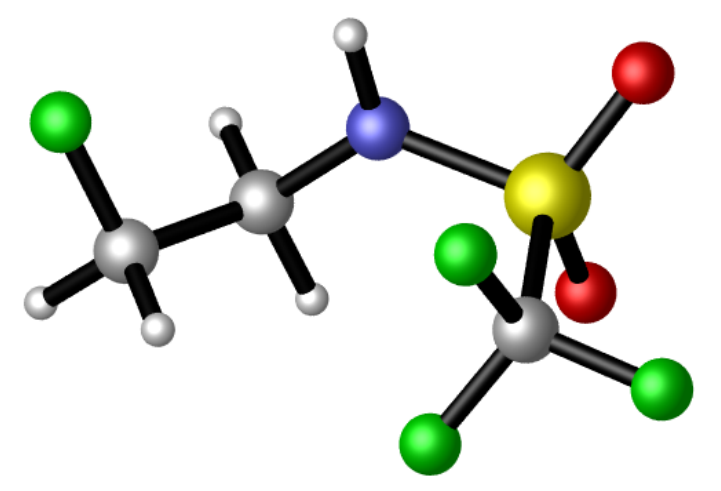

$\begin{array}{lccr}\text { S-S_3+4 } & 0.85604200 & 1.32315000 & -0.04060600 \\ \text { O-O_2 } & 1.11245800 & 1.81909800 & 1.30640800 \\ \text { O-O_2 } & 0.97686600 & 2.20058800 & -1.19487500 \\ \text { N-N_3 } & 1.84162200 & 0.01382300 & -0.38903100 \\ \text { H-H_ } & 2.64939300 & 0.34425600 & -0.91366500 \\ \text { C-C_3 } & 2.18780400 & -0.87093800 & 0.72103200 \\ \text { H-H_ } & 2.80988900 & -0.37069400 & 1.47322100 \\ \text { H-H_ } & 1.26003100 & -1.19376800 & 1.20777800 \\ \text { C-C_3 } & 2.88908900 & -2.09807700 & 0.18562300 \\ \text { H-H_ } & 3.13449700 & -2.79111000 & 0.99546500 \\ \text { H-H_ } & 2.28696500 & -2.59989200 & -0.57682000 \\ \text { F-F_ } & 4.08999100 & -1.71714000 & -0.41915200 \\ \text { C-C_3 } & -0.75217320 & 0.56034009 & -0.02776439 \\ \text { F-F_ } & -0.88148416 & -0.23533087 & -1.11067084 \\ \text { F-F_ } & -0.89050350 & -0.17921683 & 1.09313716 \\ \text { F-F_ } & -1.70424519 & 1.51703259 & -0.05602001\end{array}$

Table S10 Summary of computational Results

\begin{tabular}{ccccccc}
\hline & & \multicolumn{2}{c}{ Total Energies $^{a}$} & \multicolumn{2}{c}{ Relative Energies $^{a}$} & imaginary $^{a}$ \\
Compounds & Conformer & (a.u) & kcal/mol & (a.u) & kcal/mol & frequency \\
\hline \multirow{2}{*}{1} & Gauche & -1053.155186450 & -660865.411 & 0.000000000 & 0.00 & 0 \\
& Trans & -1053.152001760 & -660863.4126 & 0.003184690 & 2.00 & 0 \\
\hline \multirow{2}{*}{2} & Gauche & -1119.816108270 & -702695.8061 & 0.000000000 & 0.00 & 0 \\
& Trans & -1119.812805430 & -702693.7335 & 0.003302840 & 2.07 & 0 \\
\hline
\end{tabular}

${ }^{a}$ calculation performed at the wB97XD/6-31+G(d) level of theory 
The xyz coordinates for the trans conformer of 2 optimized at wB97xD/6$31+\mathrm{G}(\mathrm{d})$-levels

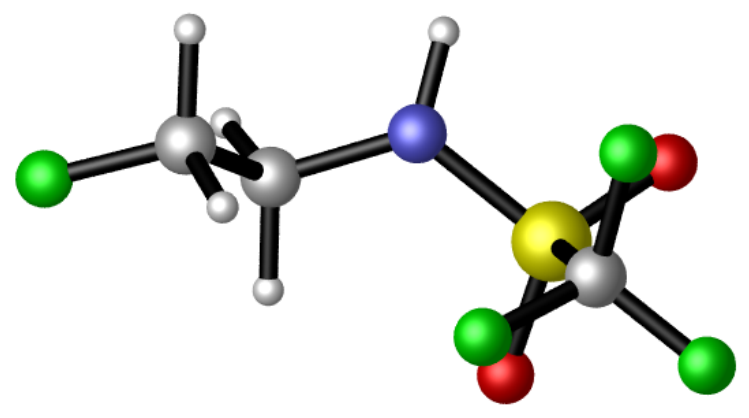

$\begin{array}{lrrr}\text { S-S_3+4 } & 0.75947400 & -0.90030400 & 0.07483800 \\ \text { O-O_2 } & 0.56509700 & -1.17474000 & 1.48102100 \\ \text { O-O_2 } & 1.65570000 & -1.64652100 & -0.78029200 \\ \text { N-N_3 } & -0.69042900 & -0.80458900 & -0.67856100 \\ \text { H-H_ } & -0.66356800 & -1.16211700 & -1.62584100 \\ \text { C-C_3 } & -1.97206700 & -0.73014800 & 0.01701800 \\ \text { H-H_ } & -2.58475700 & -1.59623300 & -0.25185800 \\ \text { H-H_ } & -1.78900400 & -0.77548800 & 1.09276400 \\ \text { C-C_3 } & -2.69511600 & 0.56072000 & -0.34463400 \\ \text { H-H_ } & -2.14670300 & 1.43657500 & 0.01160300 \\ \text { C-C_3 } & 1.35404900 & 0.86504000 & 0.00360800 \\ \text { F-F_ } & 1.44662700 & 1.26584400 & -1.26242100 \\ \text { F-F_ } & 0.48939200 & 1.65621500 & 0.64284900 \\ \text { F-F_ } & 2.54298700 & 0.95947700 & 0.58345400 \\ \text { F-F_ } & -3.94204000 & 0.55045400 & 0.26821800 \\ \text { H-H_ } & -2.85480200 & 0.64275600 & -1.42483000\end{array}$




\section{References}

1 Granger, P.; Bourdonneau, M.; Assemat, O.; Piotto, M. Concepts in Magnetic Resonance Part A 2007, 30A, 184.

2 Ackerman, J. J. H.; Soto, G. E.; Spees, W. M.; Zhu, Z. H.; Evelhoch, J. L. Magnetic Resonance in Medicine 1996, 36, 674.

Proton and Carbon NMR Spectrums 
Proton NMR Spectrum (400 MHz, DMSO)

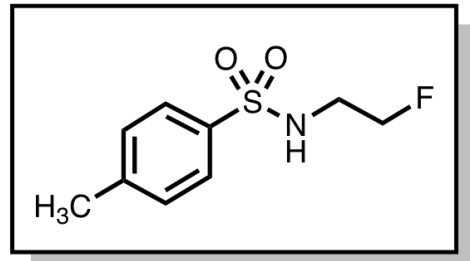

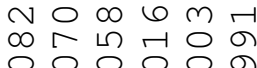

$\dot{m} \dot{m} \dot{m} \dot{m} \dot{m}$
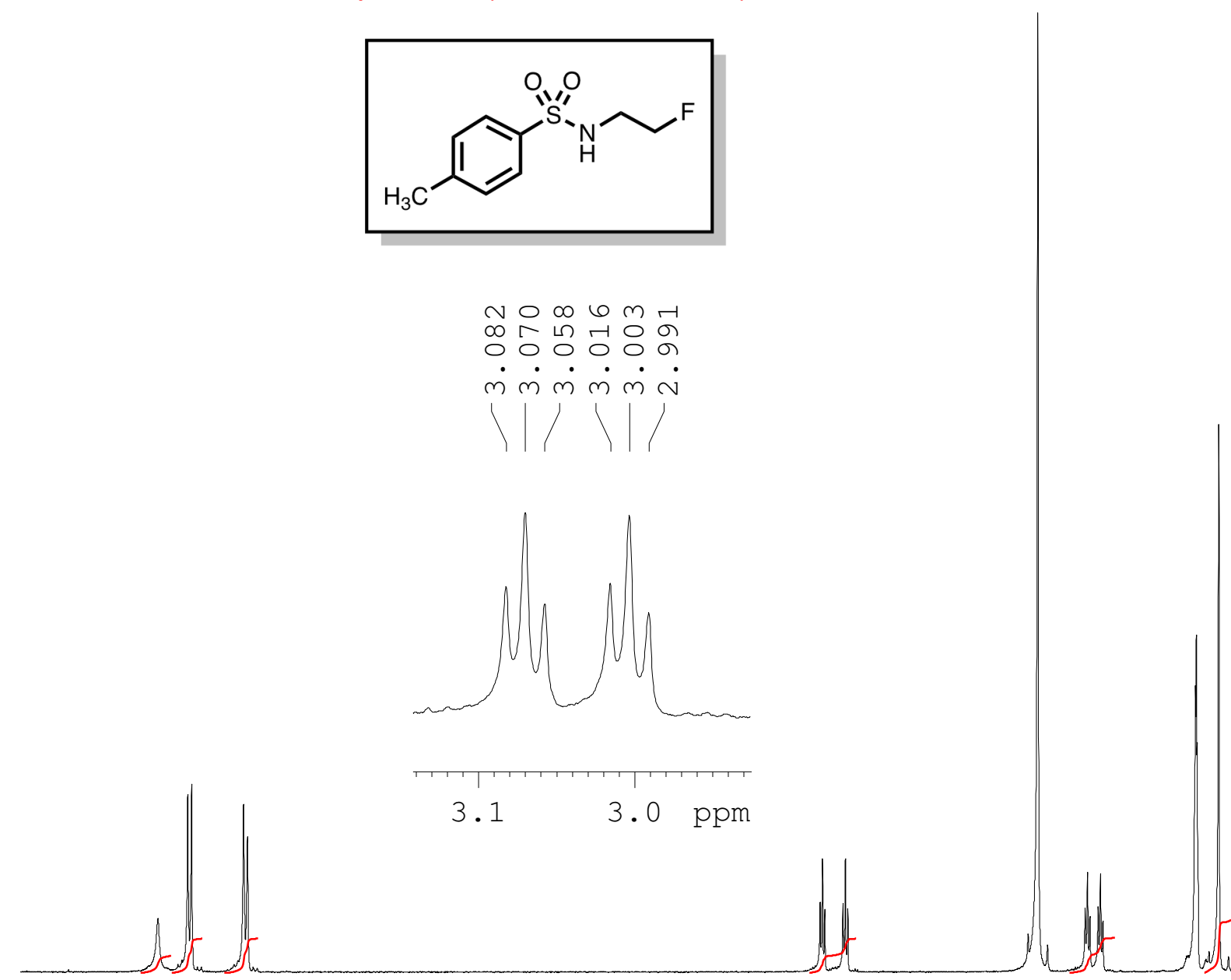

3. 1

3. 0 ppm

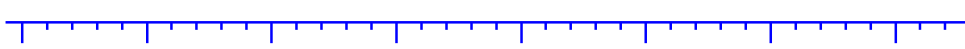

${ }^{80}$ ?

7.0

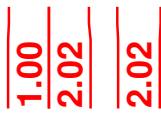

$\begin{array}{llll}6.5 & 6.0 & 5.5 & 5.0\end{array}$

4.5

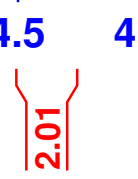

$\begin{array}{lll}4.0 & 3.5 & 3.0\end{array}$

$3.0 \quad 2.5$

52.0

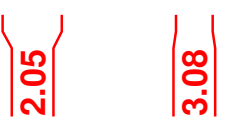

Current Data Parameters EXPNO

PROCNO

F2 - Acquisition Parameters

Date__ 20190304

20190304

Time

PROBHD

PULPROG

spect

TD

SOLVENT

NS

DS

SWH
FIDRES

AQ

RG

DW

$\mathrm{TE}$
$\mathrm{D} 1$

D1 0

$==$

SFO

NUC

1 1

PLW1

$$
\begin{array}{r}
\mathrm{zg} 30 \\
65536
\end{array}
$$

DMSO

4
2
$8012.820 \mathrm{~Hz}$ $0.122266 \mathrm{~Hz}$ $4.0894465 \mathrm{sec}$
362 62.400 use 62.400 usec
6.50 usec

$\begin{array}{rl}298.0 & \mathrm{~K} \\ 1.00000000 & \mathrm{sec} \\ 1 & \end{array}$

CHANNEL $\mathrm{f} 1========$ $400.1324710 \mathrm{MHz}$ $1 \mathrm{H}$
9.30 use $18.00000000 \mathrm{~W}$

F2 - Processing parameters SI 65536

$\begin{array}{lc}\text { SF } & 400.1300000 \mathrm{MHz} \\ \text { WDW } & \text { EM }\end{array}$

SSB

LB

GB
PC

EM

$0.30 \mathrm{~Hz}$

1.00 
Current Data Parameters

F-CH2-CH2-NTs-anion-kotbu

PROCNO

F2 - Acquisition Parameters

$\begin{array}{lr}\text { Date } & 20190304 \\ \text { Time } & 17.12\end{array}$

spect
INSTRUM
PROBHD $5 \mathrm{~mm}$ PABBO BB-

PUTPOG

TD SOLVENT

Proton NMR Spectrum (400 MHz, DMSO)

NS

SWH

$8012.820 \mathrm{~Hz}$

$0.122266 \mathrm{~Hz}$
$\mathrm{H}$$\quad 4.0894465 \mathrm{sec}$

$\begin{array}{lr}\text { RG } & 256 \\ \text { DW } & 62.400 \text { usec }\end{array}$

$\begin{array}{lr}\text { DW } & 62.400 \text { use } \\ \text { DE } & 6.50 \text { usec }\end{array}$

$\begin{array}{lr}\text { TE } & 298.0 \mathrm{~K} \\ \text { D1 } & 1.00000000 \mathrm{sec}\end{array}$

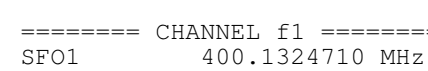

$\begin{array}{lr}\text { SEO1 } & 400.1324710 \mathrm{MHz} \\ \text { NUC1 } & 1 \mathrm{H}\end{array}$

$\begin{array}{lr}\text { P1 } & 9.30 \text { usec } \\ \text { PLW1 } & 18.00000000 \mathrm{~W}\end{array}$

F2 - Processing parameters

$\begin{array}{lc}\text { SF } & 400.1300000 \mathrm{MHz} \\ \text { WDW } & \text { EM }\end{array}$

$\begin{array}{lll}\text { SSB } & 0 & 0.30 \mathrm{~Hz}\end{array}$

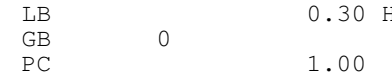

の $\begin{array}{lllll}m & 6 & m & 6 & \circ \\ \infty & 6 & n & m & \sim\end{array}$

$\infty \begin{array}{llllll}\infty & \infty & \infty & \infty & \infty & \infty\end{array}$

$\dot{\sim} \dot{\sim} \sim \dot{\sim} \dot{\sim}$

$1|1|$
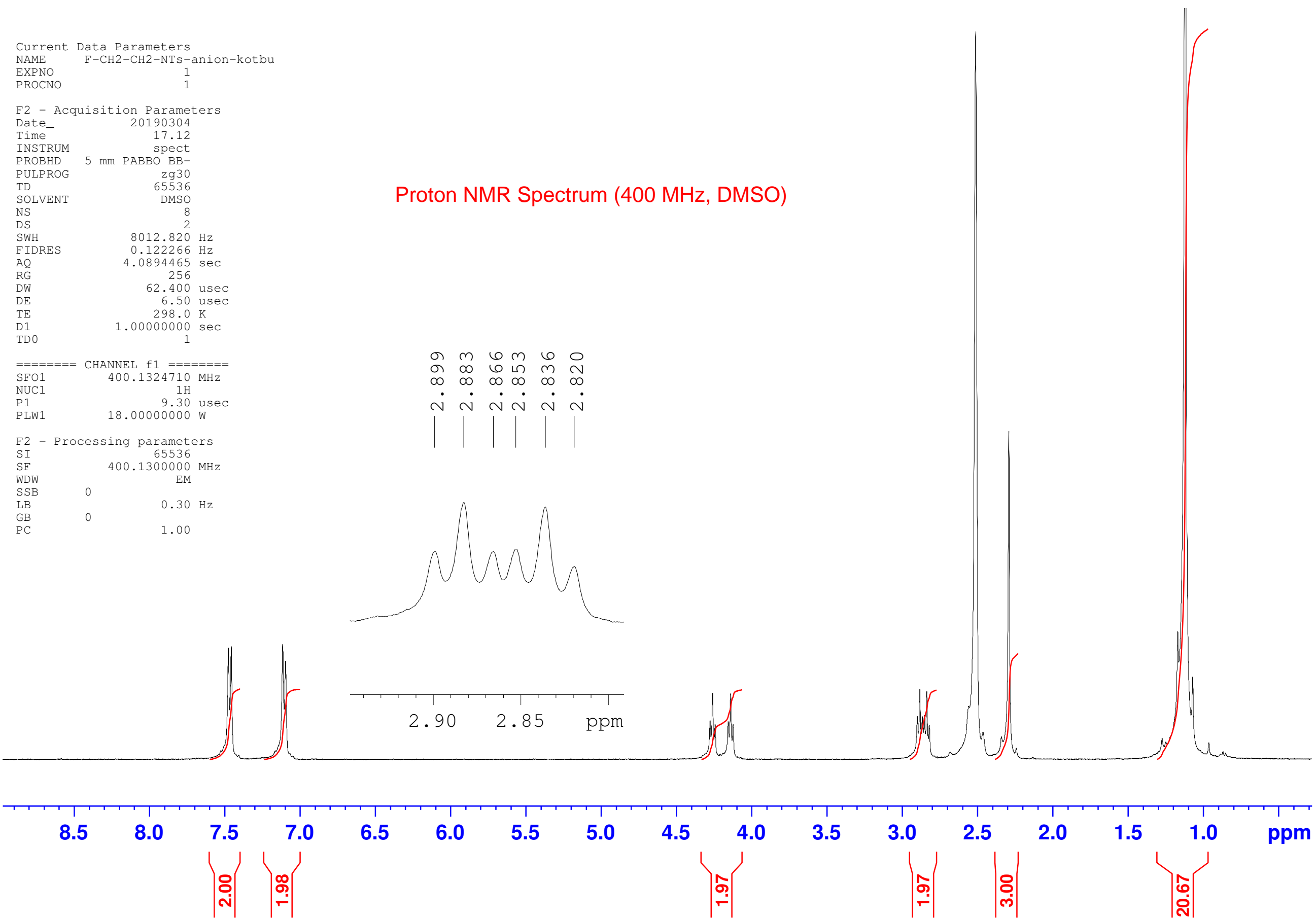

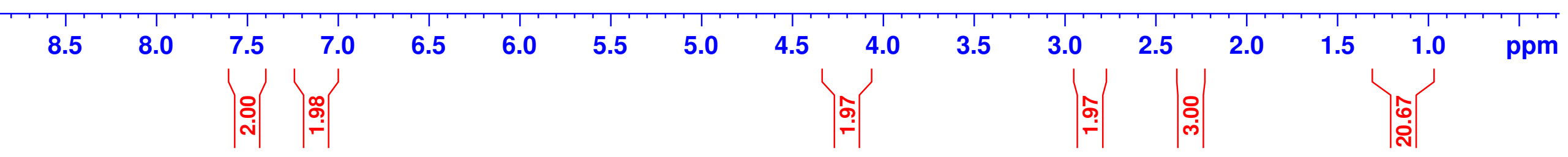


Carbon NMR Spectrum (400 MHz, DMSO)

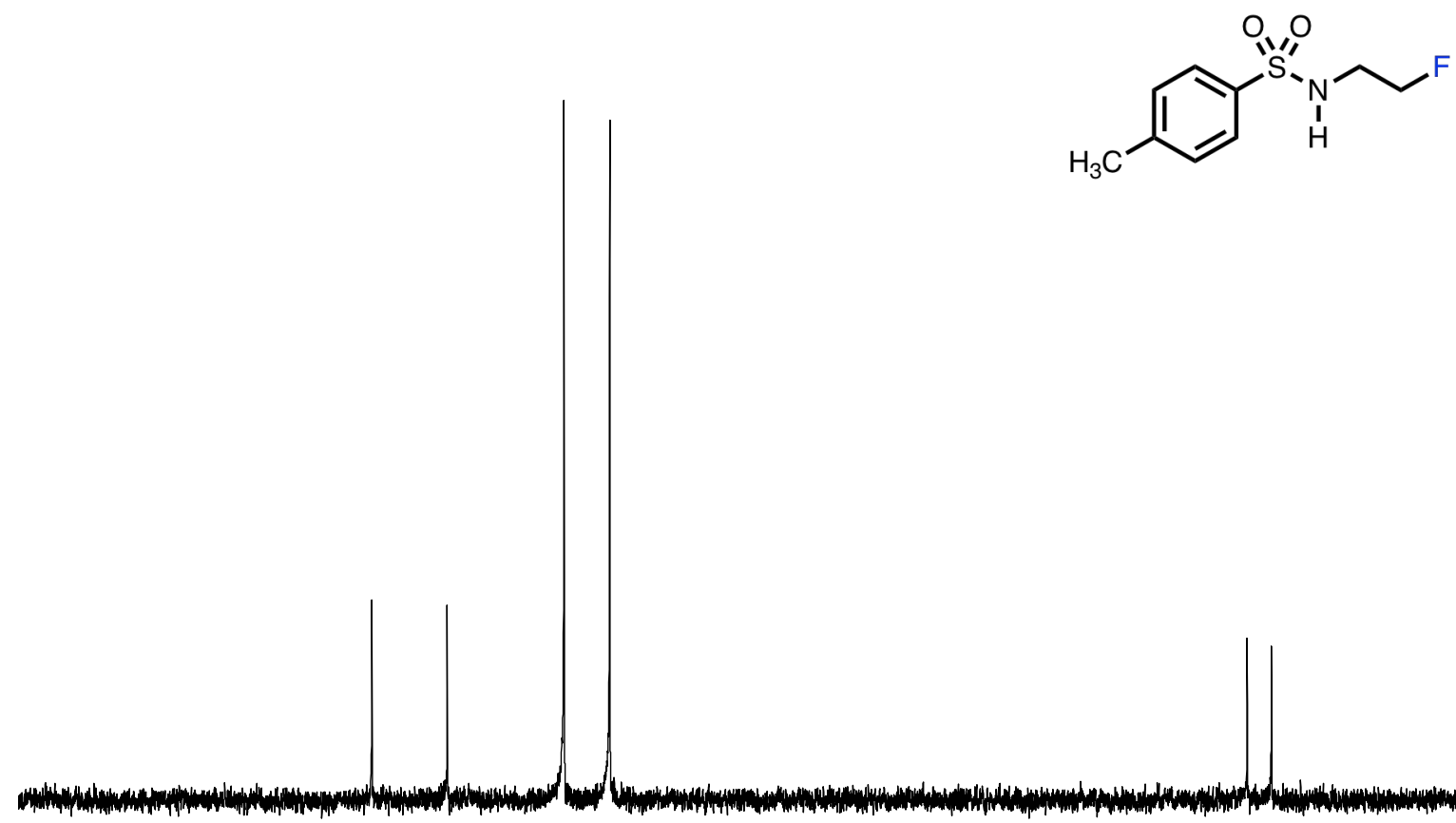



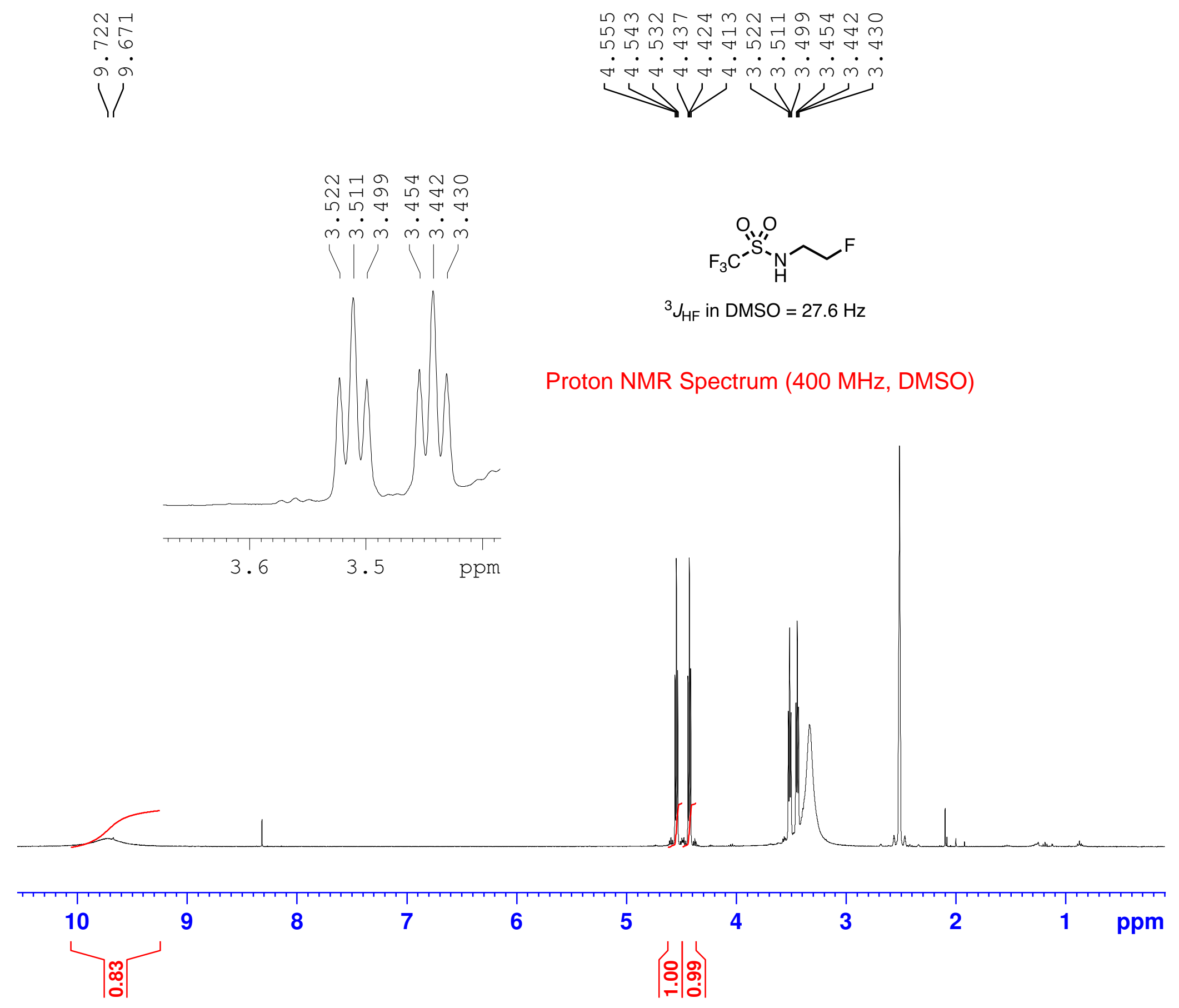

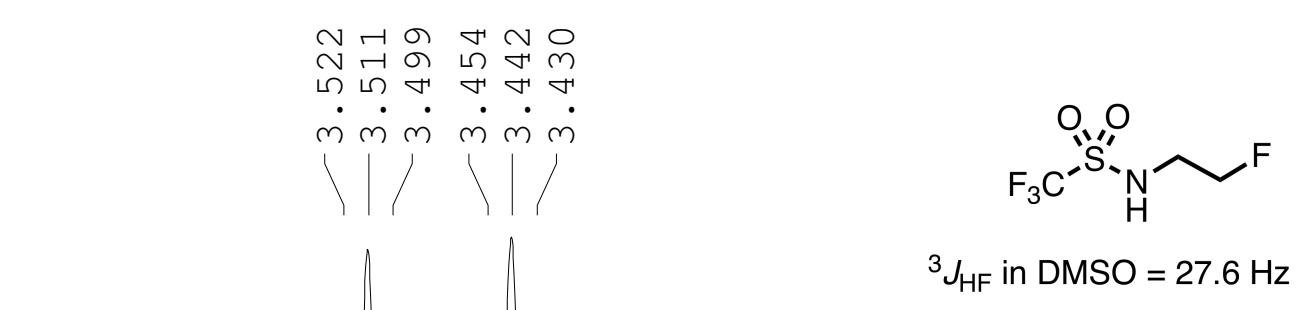

Proton NMR Spectrum (400 MHz, DMSO)

๓m

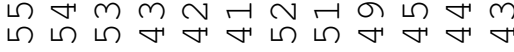

$\dot{\forall} \dot{\gamma} \dot{\gamma} \dot{\gamma} \dot{m} \dot{m} \dot{m} \dot{m} \dot{m}$

$\rightarrow$

${ }^{3} J_{\mathrm{HF}}$ in $\mathrm{DMSO}=27.6 \mathrm{~Hz}$

Proton NMR Spectrum (400 MHz, DMSO)

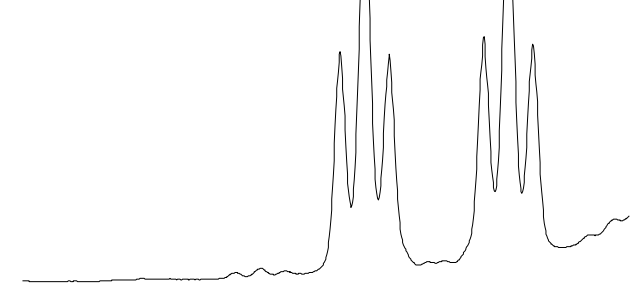

Current Data Parameters

NAME

EXPNO

F2 - Acquisition Parameters

Date_ 20190320

Time $\quad 15.56$

INSTRUM spect

PROBHD $5 \mathrm{~mm}$ PABBO BB-

$\begin{array}{lr}\text { PULPROG } & \text { zg30 } \\ \text { TD } & 65536\end{array}$

SOLVENT DMSO

NS

DS

SWH

FIDRES

AQ

DW

$\mathrm{DE}$

D1

TD 0

SFO1

NUC1

P 1

16
2
$8012.820 \mathrm{~Hz}$
$0.122266 \mathrm{~Hz}$ $4.0894465 \mathrm{sec}$

645 usec

6.50 usec

$1.00000000 \mathrm{sec}$

$400.1324710 \mathrm{MHz}$

9.30 usec

F2 - Processing parameters

SI 65536

$400.1300000 \mathrm{MHz}$

$\begin{array}{lll}V & E M \\ B S B & 0 & 0.30 \mathrm{~Hz}\end{array}$

$\begin{array}{lll} & 0 & 0.30 \\ \mathrm{~GB} & 0\end{array}$ 


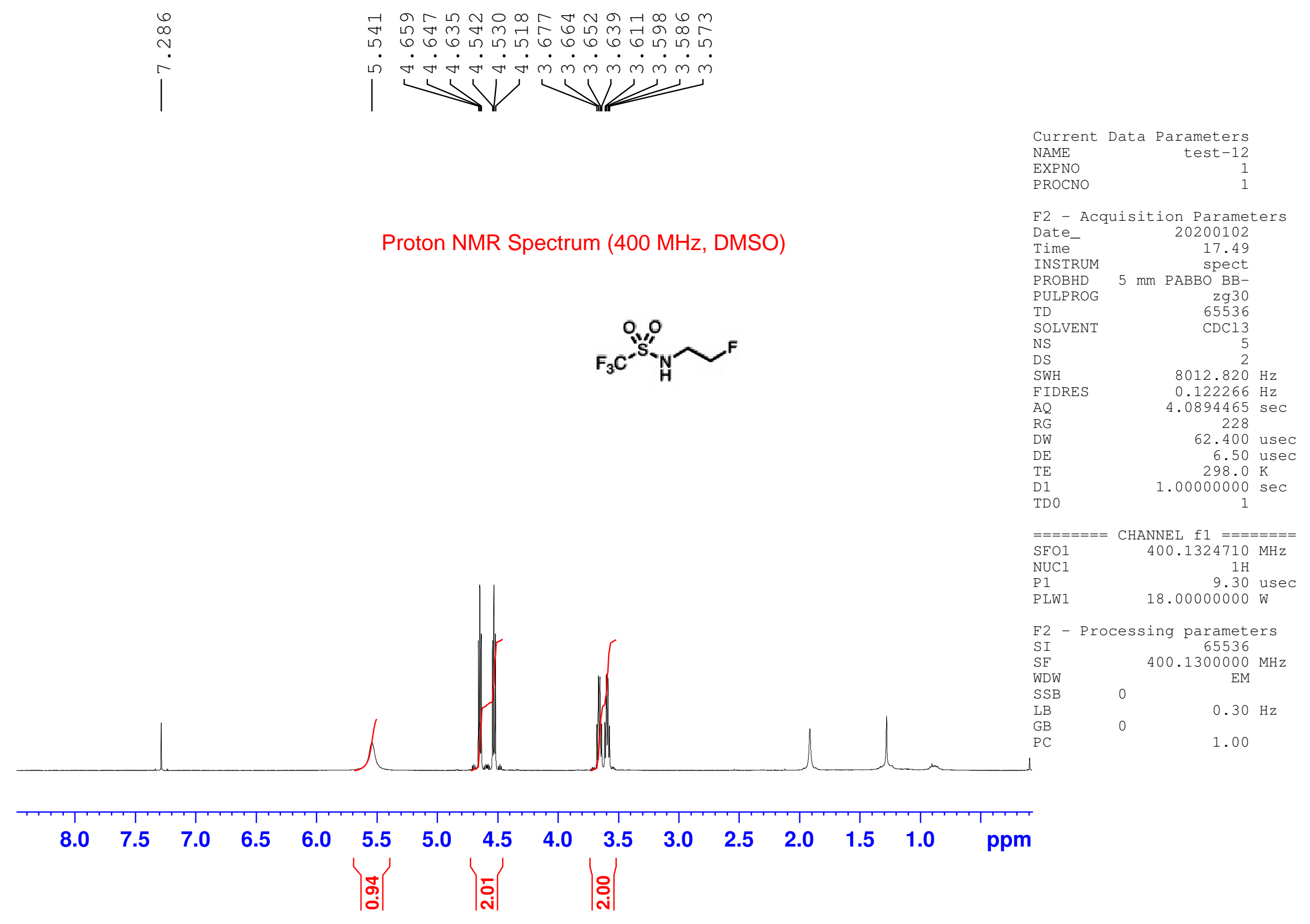




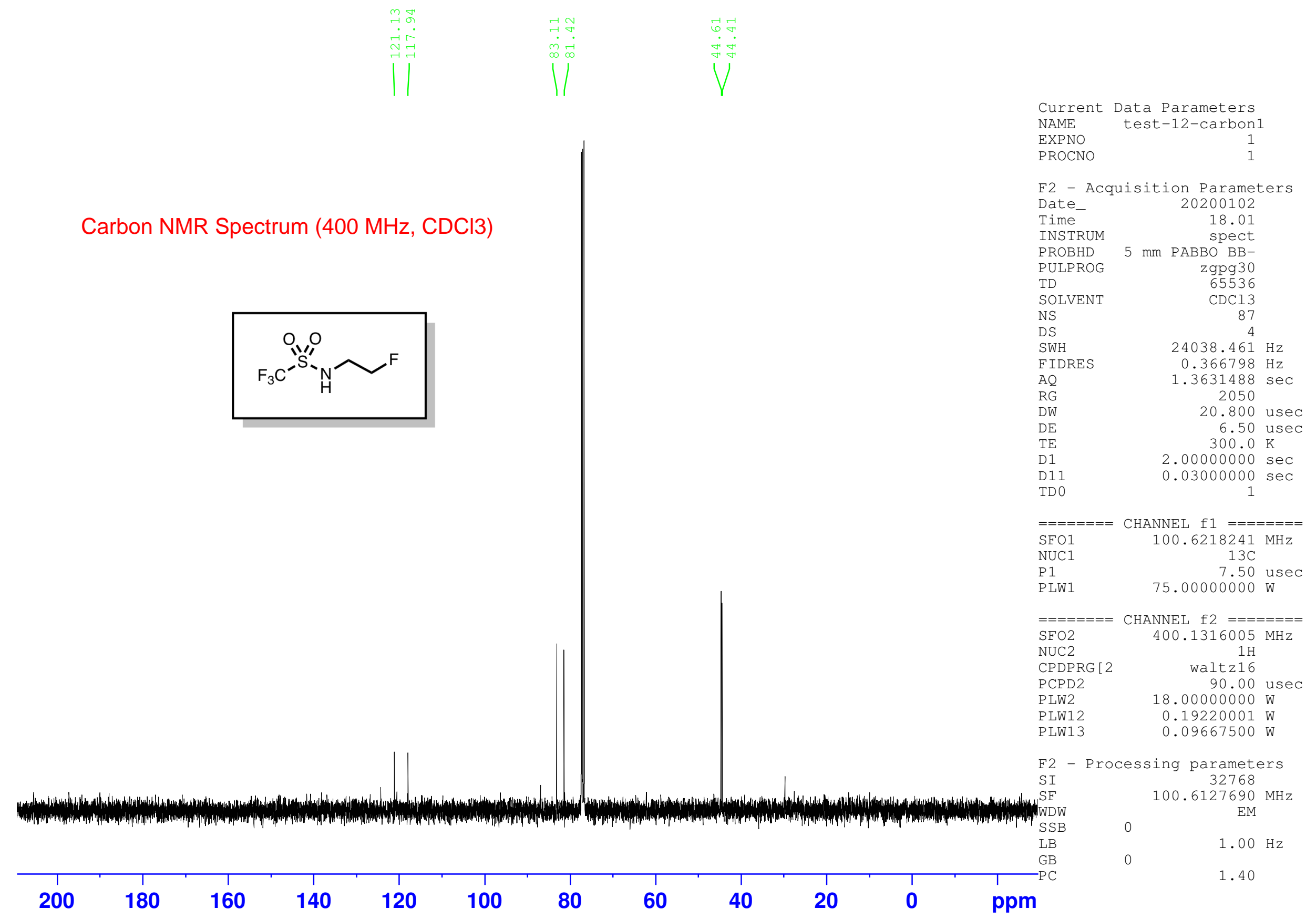




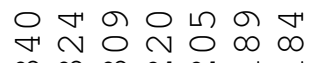

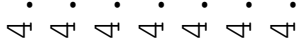

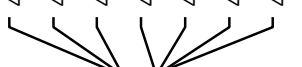

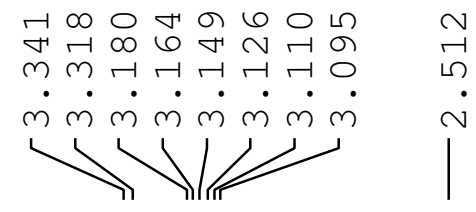

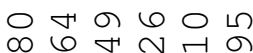

Proton NMR Spectrum (400 MHz, DMSO)

म

$\dot{m} \dot{m} \dot{m} \dot{m} \dot{m}$

1111
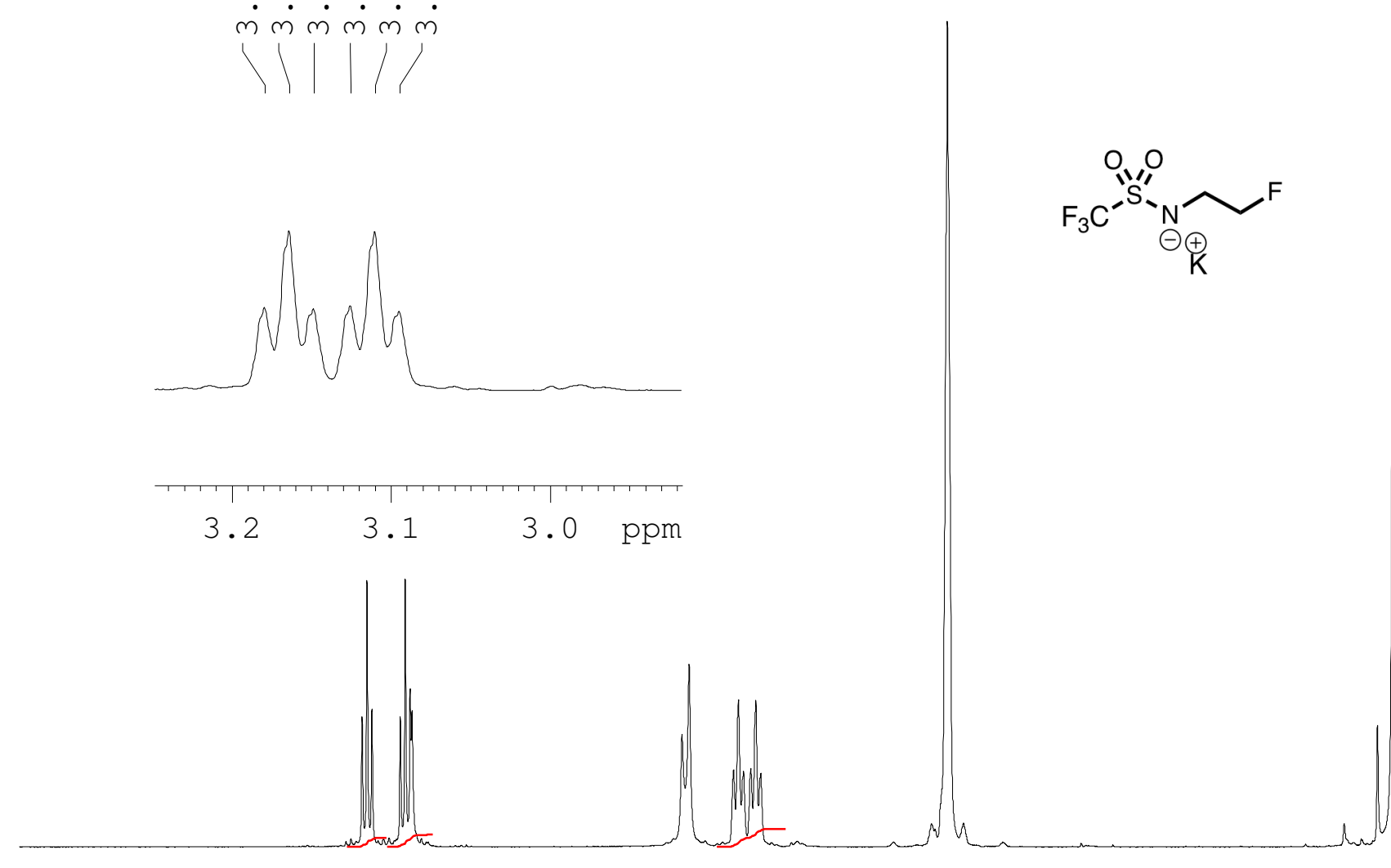

Current Data Parameters
NAME
F-CH2-CH2-NTf-anion-ktoBU3

EXPNO

ROCNO

F2 - Acquisition Parameters

$\begin{array}{lr}\text { Date } & 20190320 \\ \text { Time } & 16.31\end{array}$

INSTRUM spect

PULPROG

SOLVENT

NS
DS

$\begin{array}{ll}2 \\ \text { SWH } & 8012.820 \mathrm{~Hz}\end{array}$

$0.122266 \mathrm{~Hz}$
$4.0894465 \mathrm{sec}$

RG

DW

$\mathrm{DE}$
$\mathrm{TE}$
$\mathrm{D} 1$

$4.0894465 \mathrm{sec}$
$256 \mathrm{~S}$

62.400 usec

D1

$\begin{array}{rl}298.0 & \mathrm{~K} \\ 1.00000000 & \mathrm{sec}\end{array}$

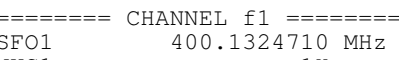

$\begin{array}{lr}\text { SEO1 } & 400.1324710 \mathrm{MHz} \\ \text { NUC1 } & 1 \mathrm{H}\end{array}$

$\begin{array}{ll}\text { P1 } & 9.30 \mathrm{usec} \\ \text { PLW1 } & 18.00000000 \mathrm{~W}\end{array}$

F2 - Processing parameters

SI $\quad 400.1300000 \mathrm{MHz}$

WDW $\quad 400.1300000$

SSB

$0.30 \mathrm{~Hz}$

1.00

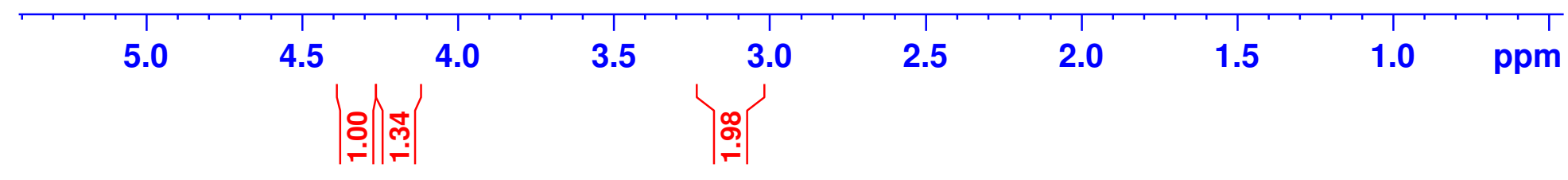


Current Data Parameters
NAME
F-CH2-CH2-NTf

EXPNO
PROCNO
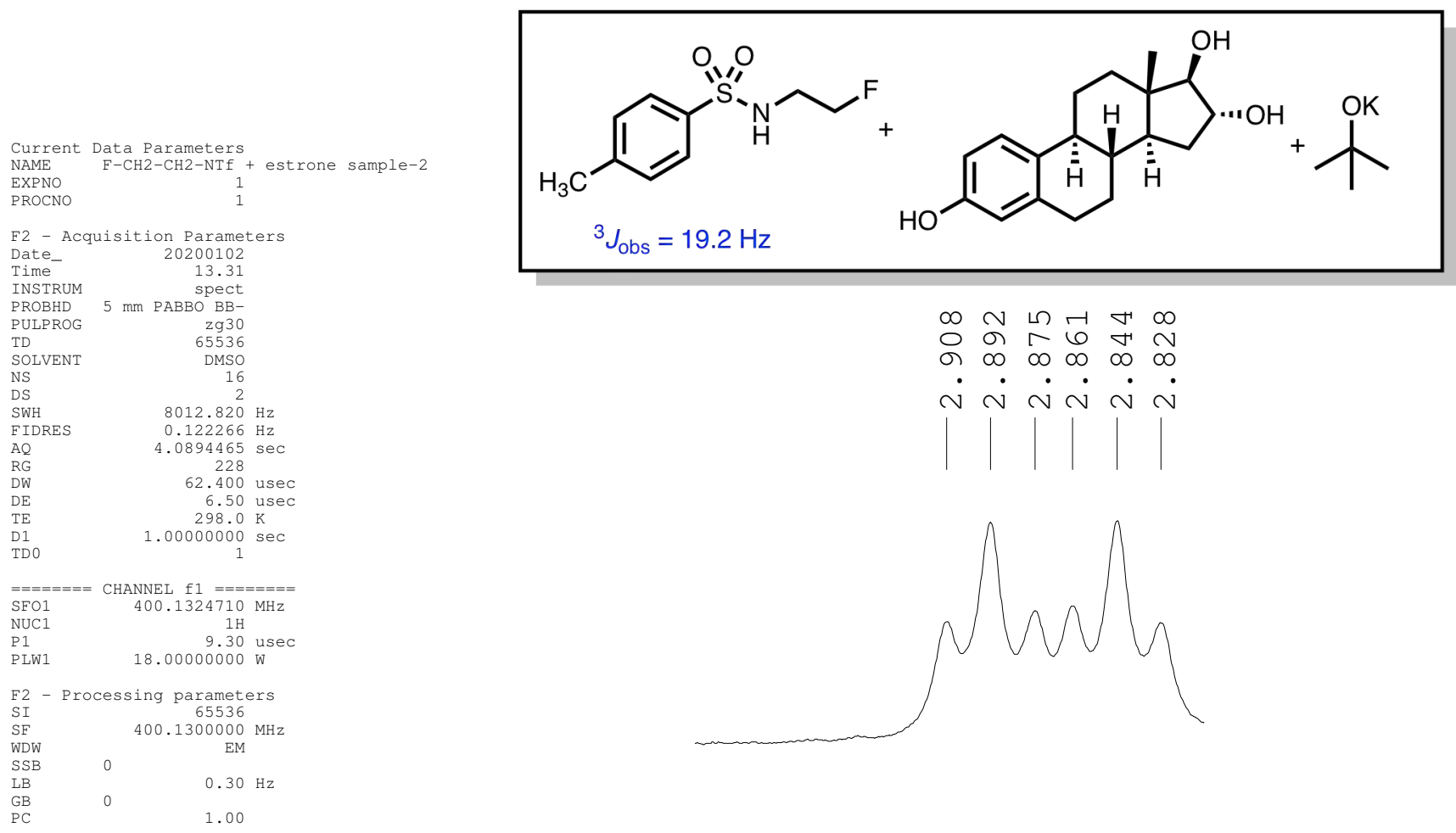

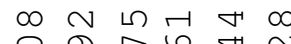

न $\infty \infty \infty \infty$

$\sim \sim \sim \sim \sim$
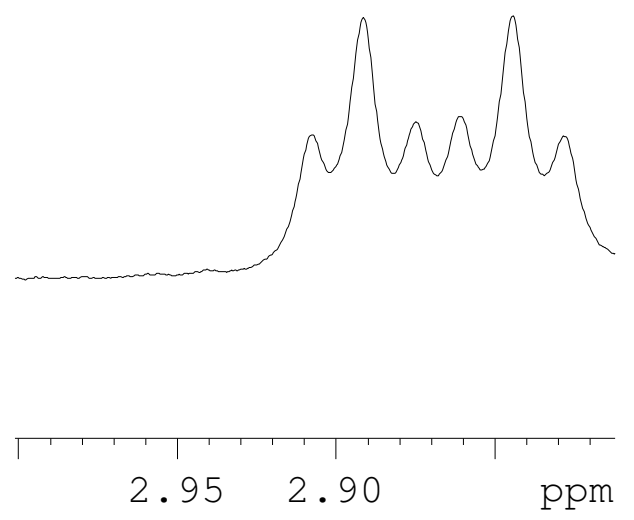

Proton NMR Spectrum (400 MHz, DMSO)

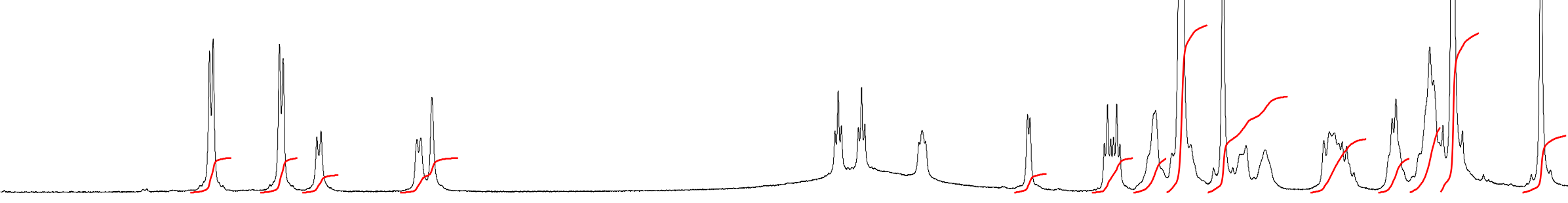

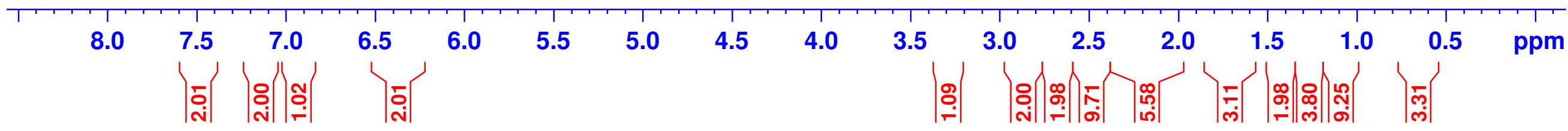




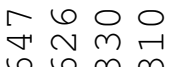
$66 \mathrm{~m}$
VV

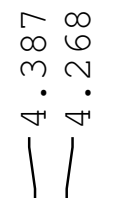
m담
ㅂํㅇํำ
$m \sim \sim \sim \sim$
$1 / 111$

Proton NMR Spectrum (400 MHz, DMSO)
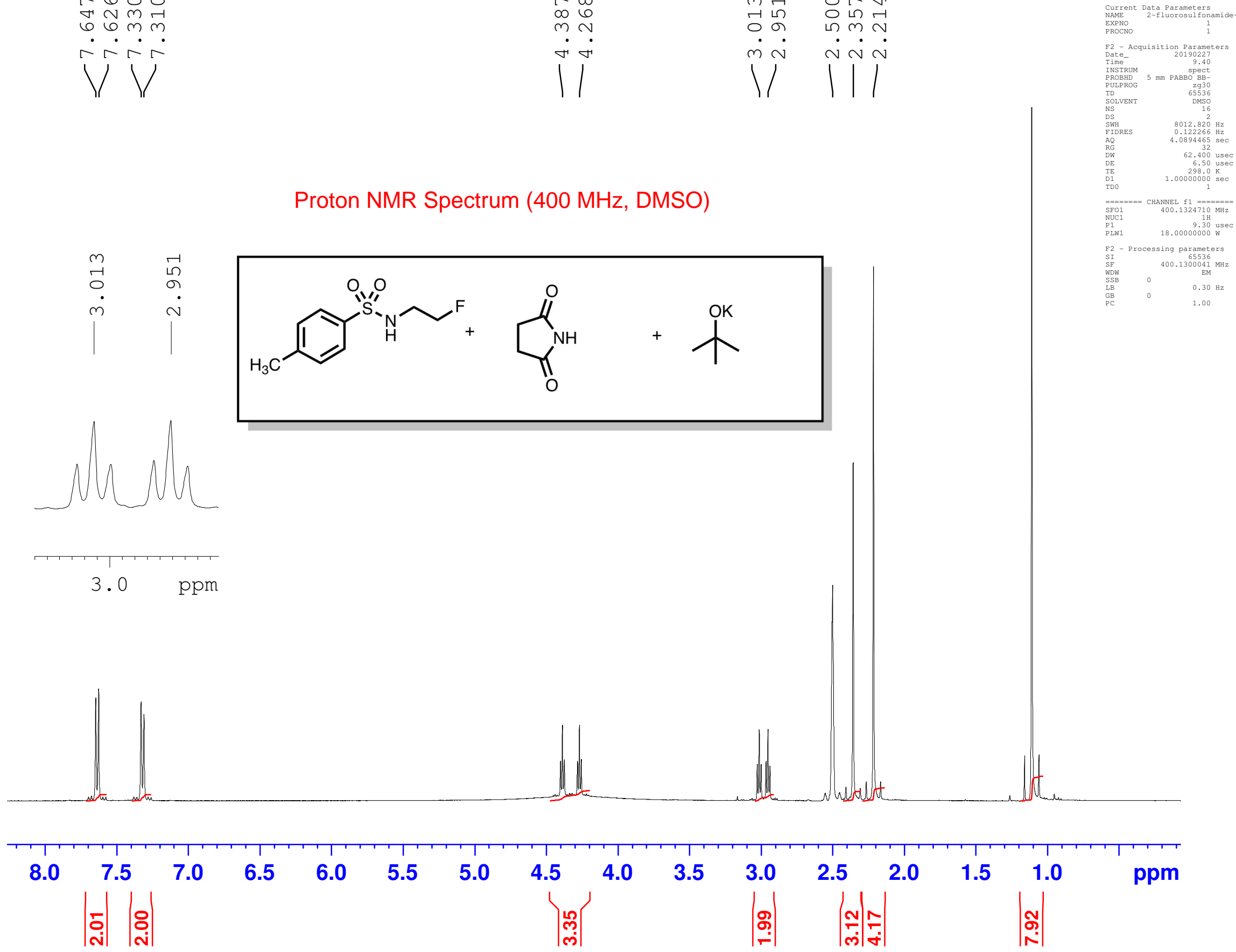
Proton NMR Spectrum (400 MHz, DMSO)
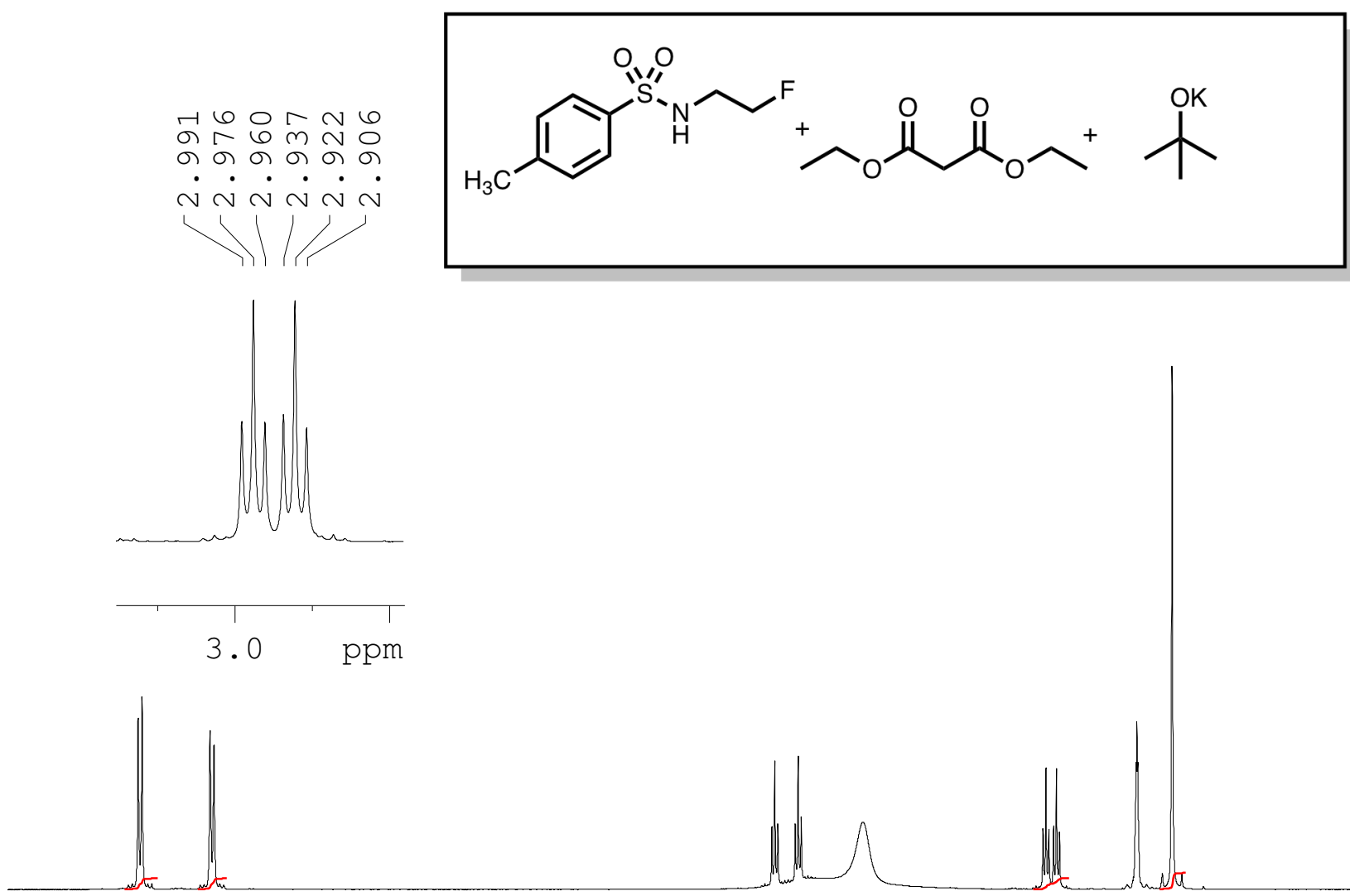

pm
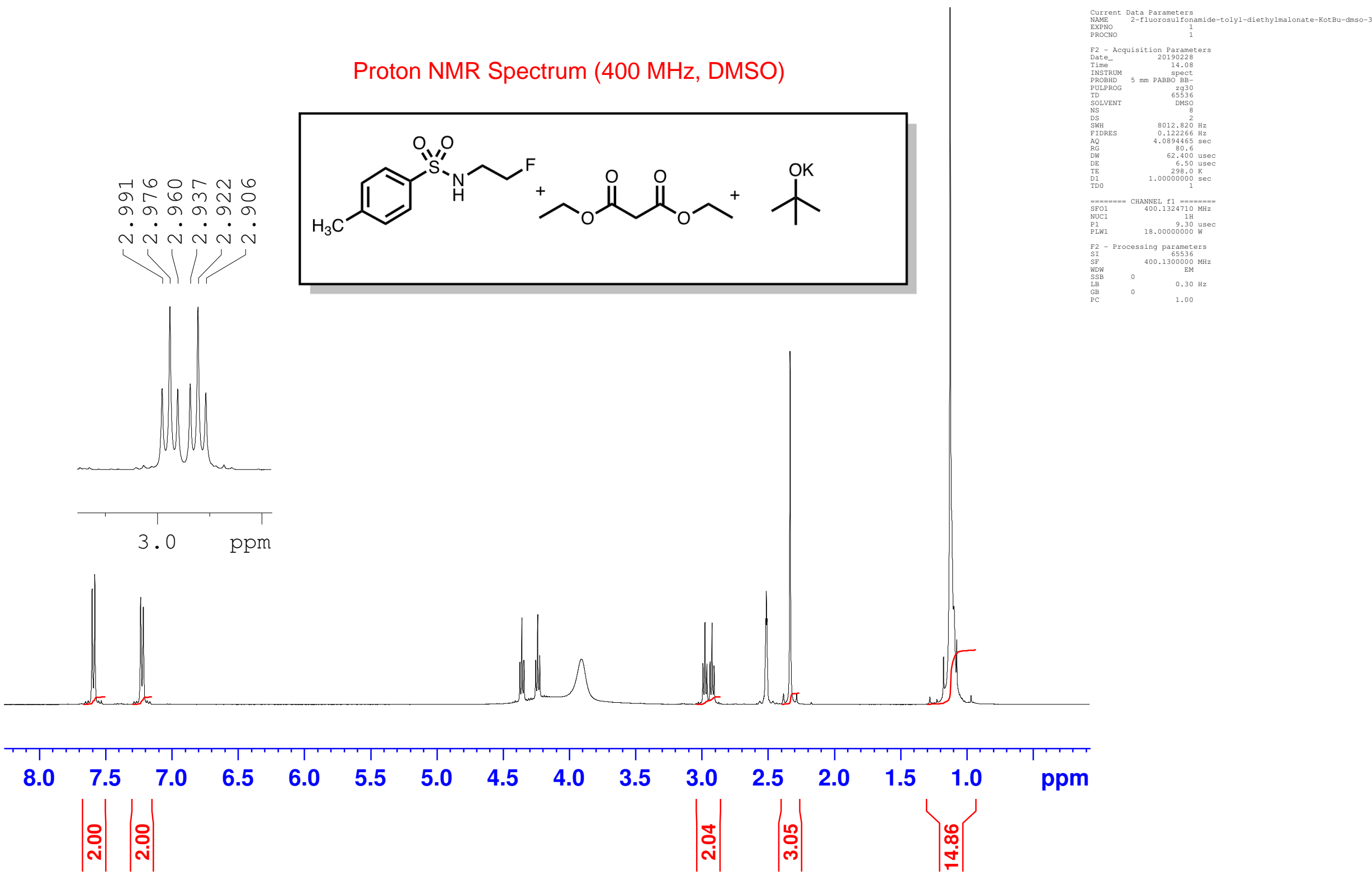
Proton NMR Spectrum (400 MHz, DMSO)
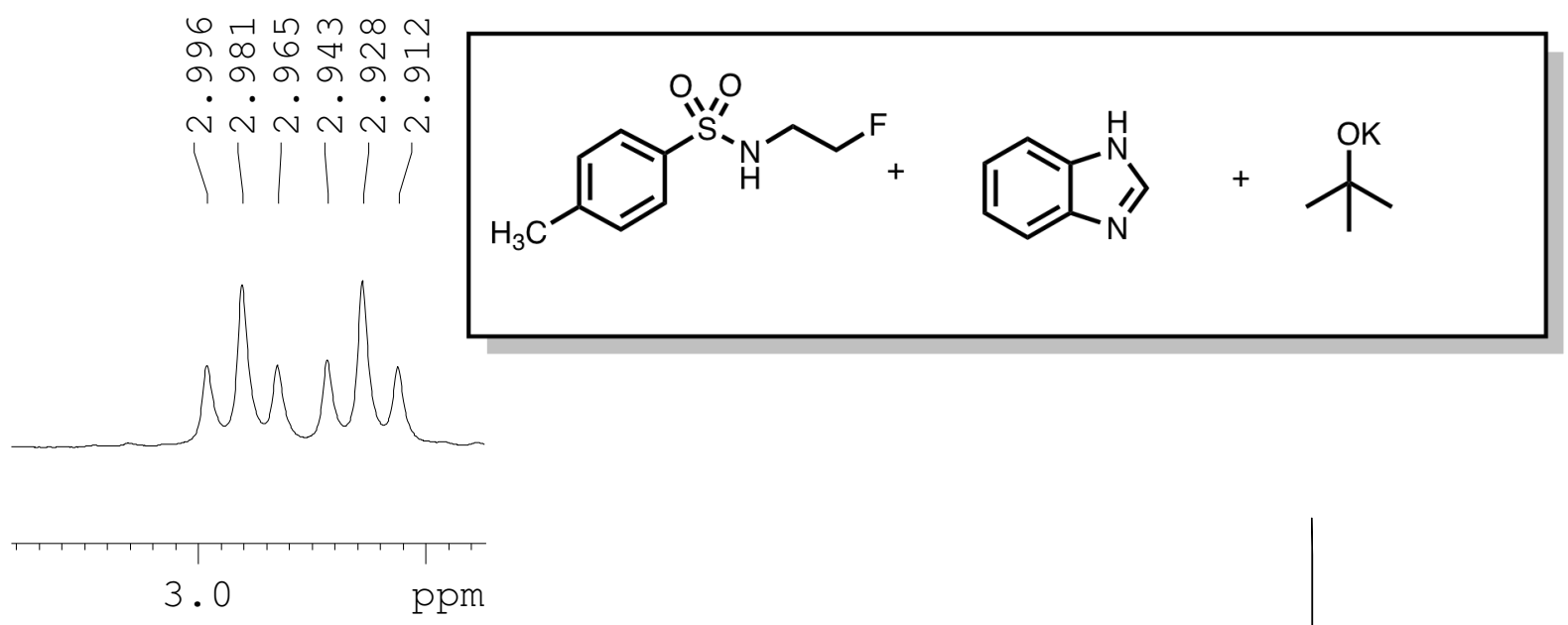

om
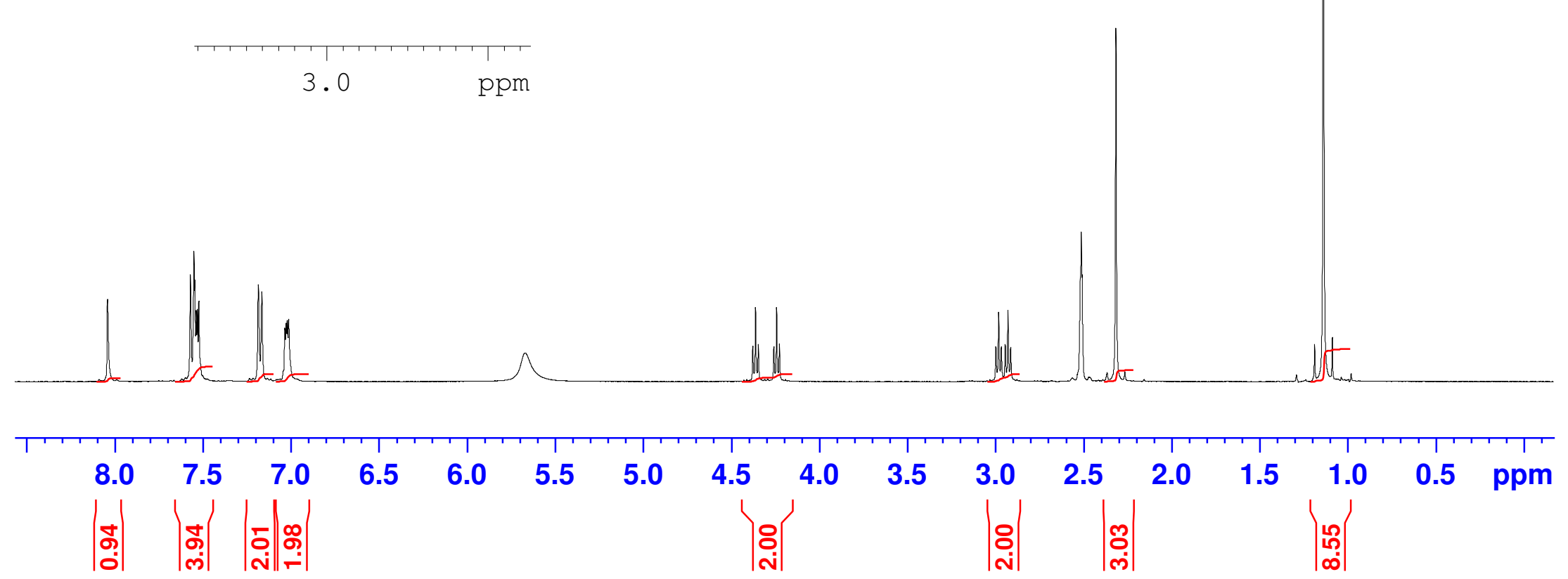


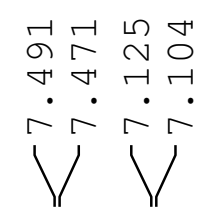

$6 \circ m \curvearrowleft \sim 6$

ด

$\dot{\sim} \dot{\sim} \dot{\sim} \dot{\sim} \dot{\sim}$
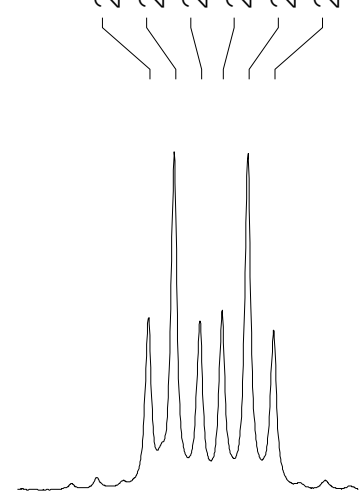

2.9 ppm a) $\backsim \cap m \in$

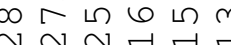

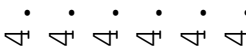

$>1$

Proton NMR Spectrum (400 MHz, DMSO)
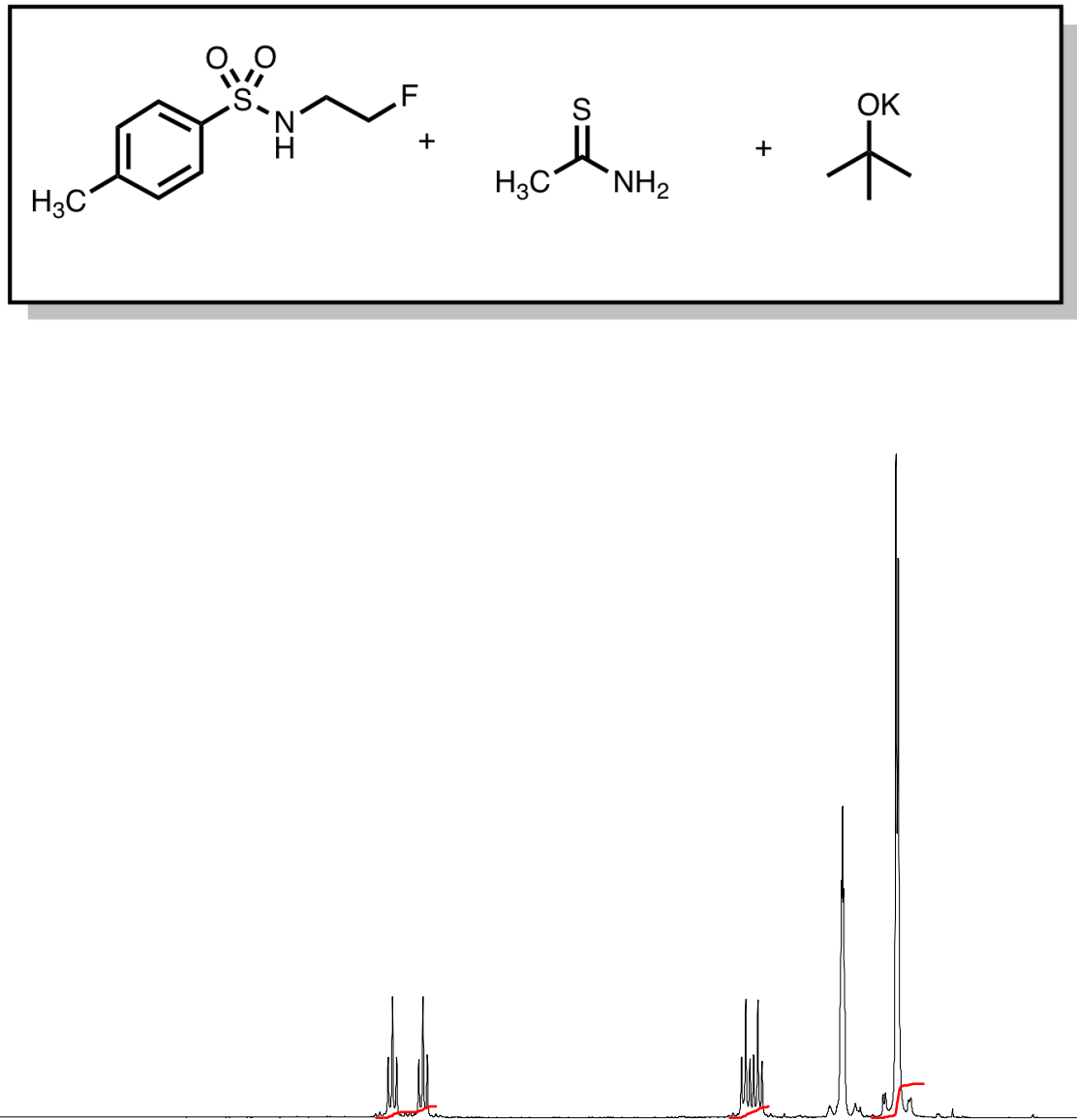

Current Data Parameters $\circ$ a

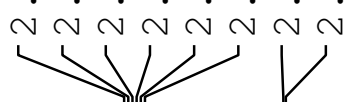

EXPNO
PROCNO

F2 - Acquisition Parameter

Date 20190405

spect
INROBHD
PROBH $5 \mathrm{~mm}$ PABBO BB-

PUTPROC

TD SOLVENT

NS
DS
SWH

$\begin{array}{ll}\text { FIDRES } & 8012.820 \mathrm{~Hz} \\ \text { AQ } & 0.122266 \mathrm{~Hz} \\ \text { R } & 4.0894465 \mathrm{sec}\end{array}$

$\begin{array}{lr}\text { RG } & 228 \\ \text { DW } & 62.400 \text { usec } \\ \text { DE } & 6.50 \text { usec }\end{array}$

$\begin{aligned} 6.50 & \text { use } \\ 298.0 \mathrm{~K} & \end{aligned}$

$\underset{T D O}{D 1}$

$=======$ CHANNEL $\mathrm{f} 1 \mathrm{I}=======$
SFO1
$400.1324710 \mathrm{MHz}$

NUC1

$18.00000000 \mathrm{~W}$

F2 - Processing paraneters

F2 - Process parameters

$\begin{array}{ll}\text { SE } & 400.1300000 \mathrm{MH} \\ \text { WDW } & \end{array}$

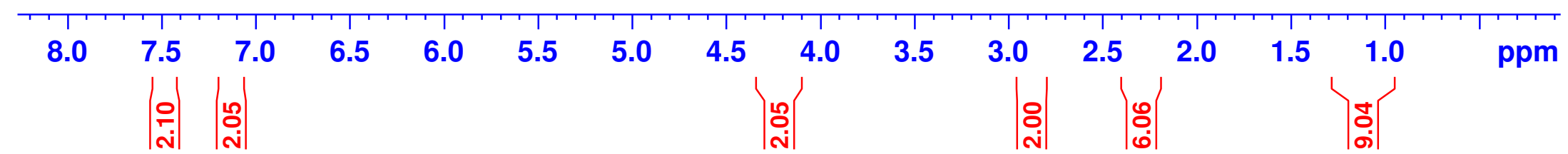


Proton NMR Spectrum (400 MHz, DMSO)
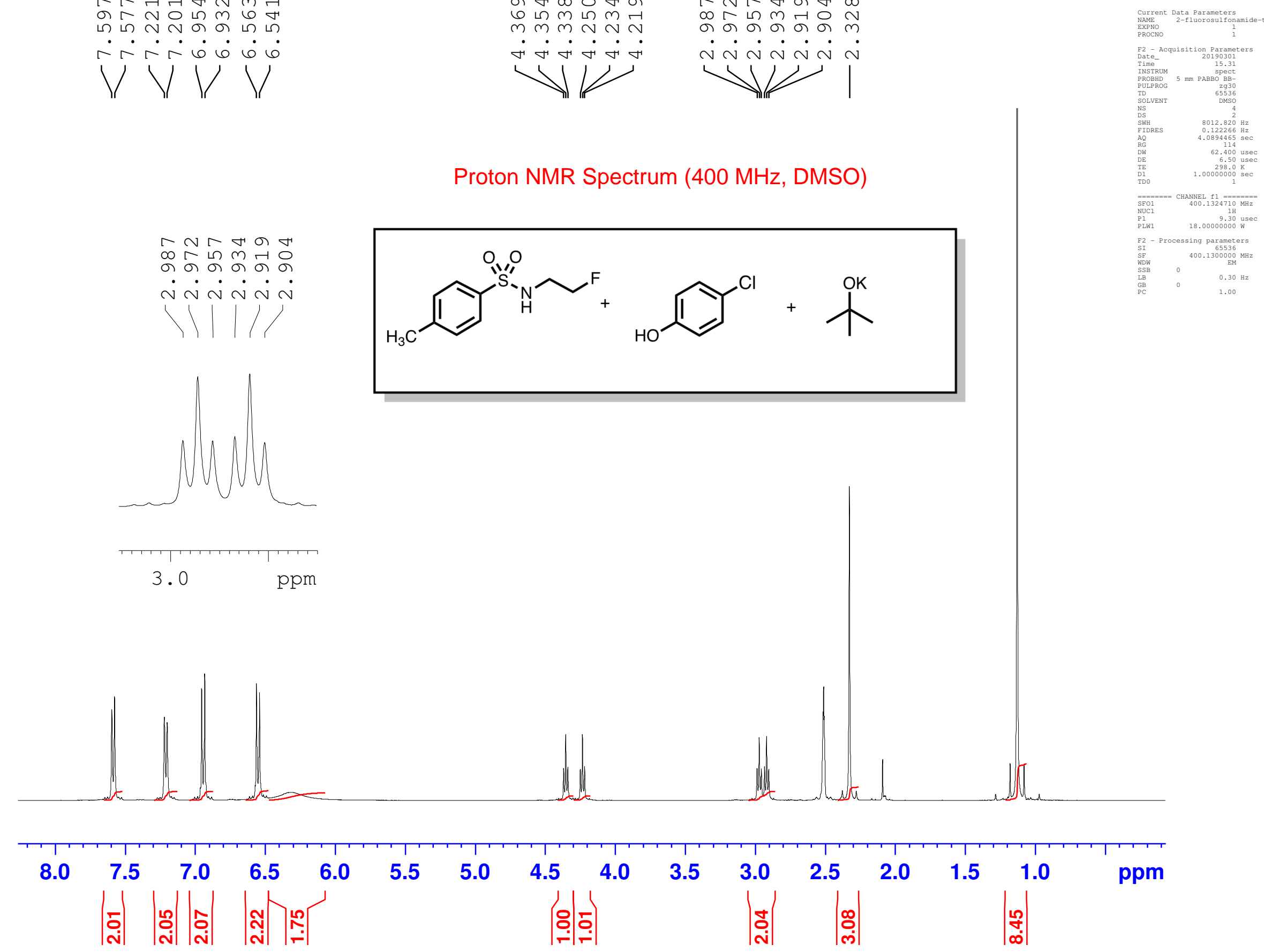


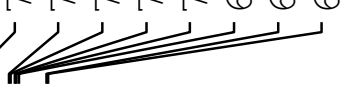

Proton NMR Spectrum (400 MHz, DMSO)
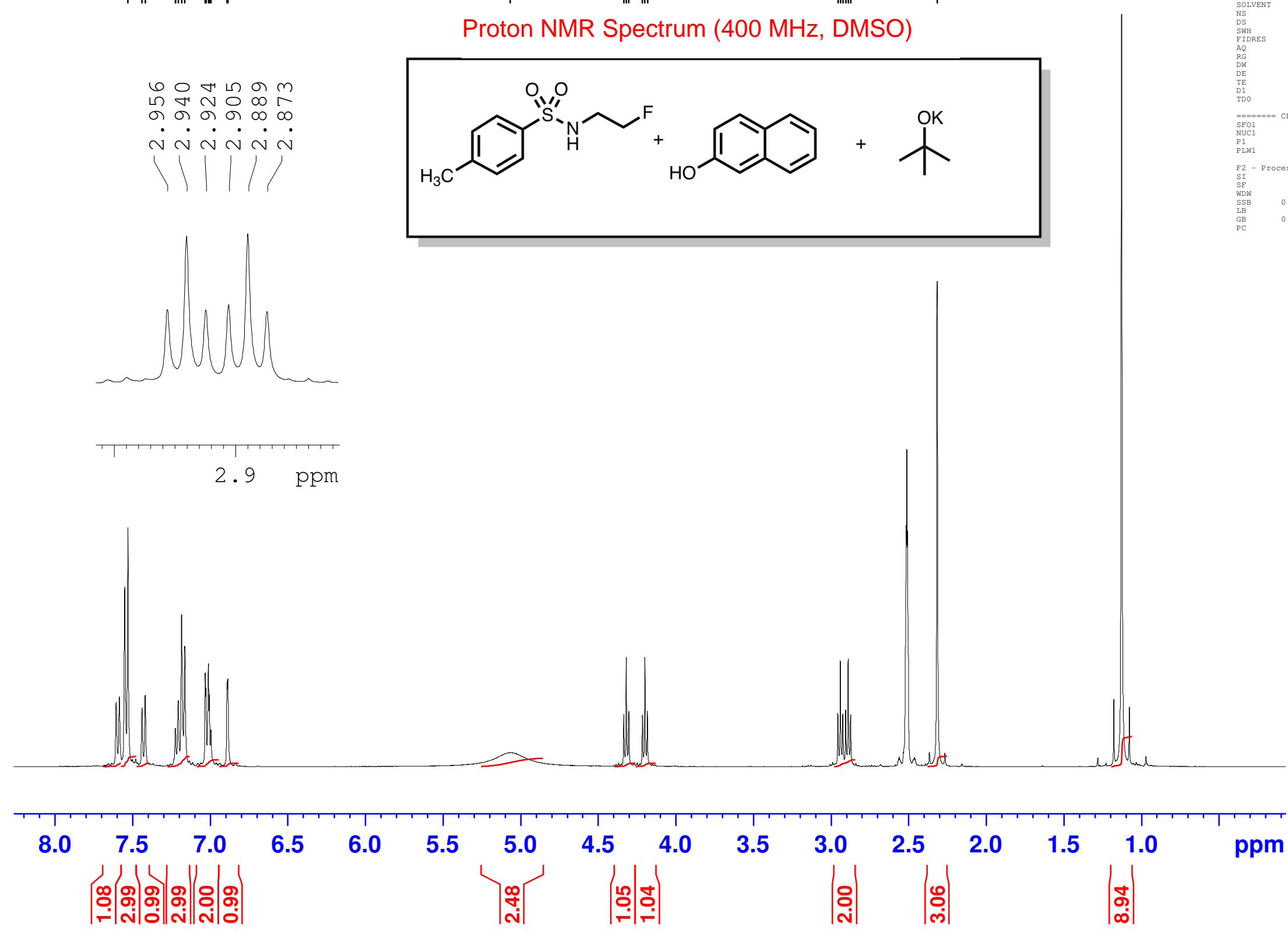

. . . . . . . 
Proton NMR Spectrum (400 MHz, DMSO)

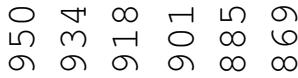
$\dot{\sim} \dot{\sim} \dot{\sim} \dot{\sim} \dot{\sim}$
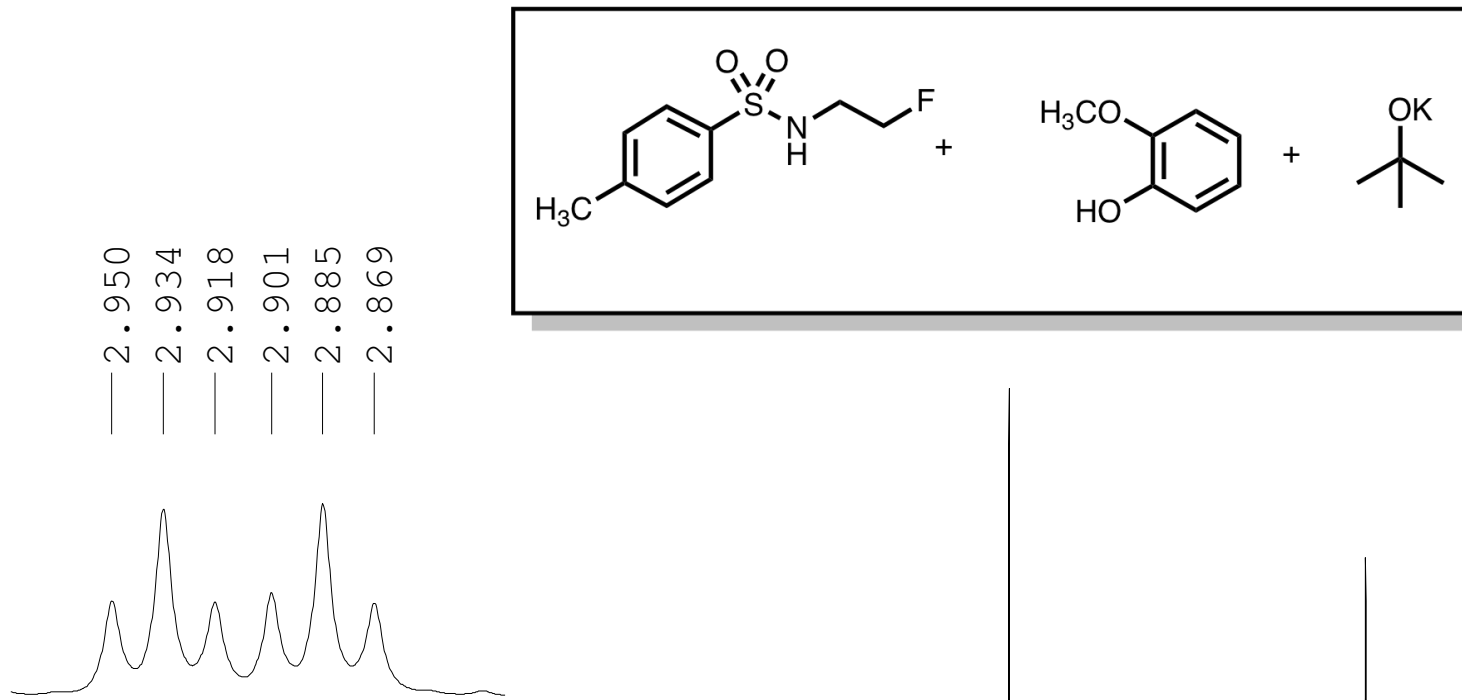

$\sin n \sim$
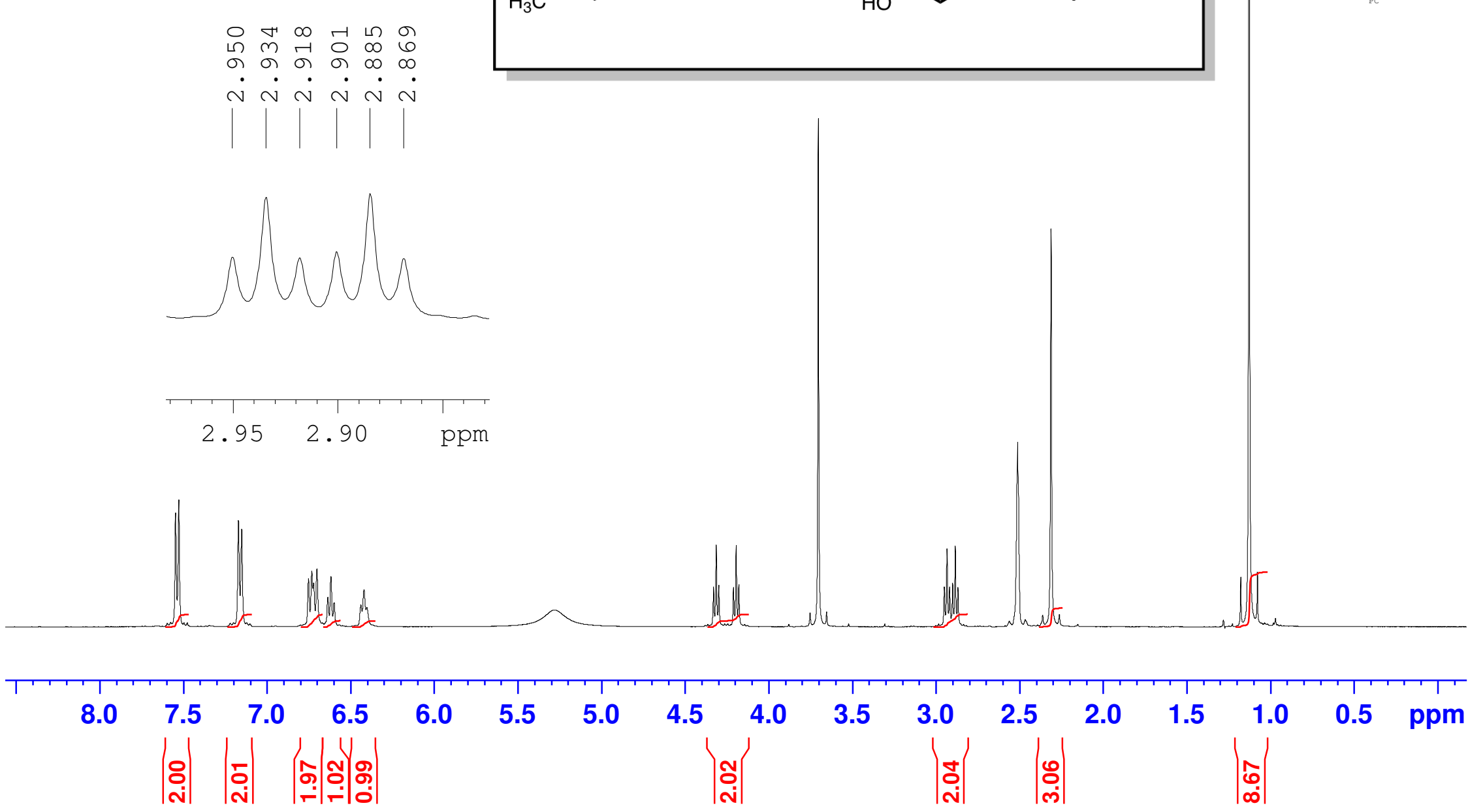
の भr

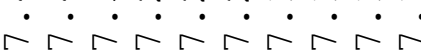

$\rightarrow$

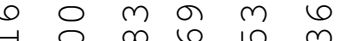

ने

$\dot{\sim} \dot{\sim} \dot{\sim} \dot{\sim} \dot{\sim}$
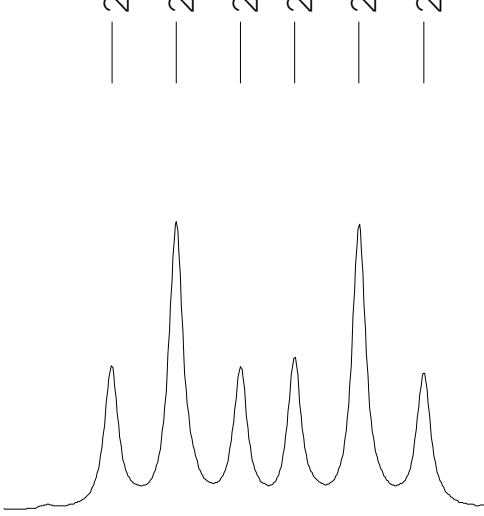

2.90

ppm

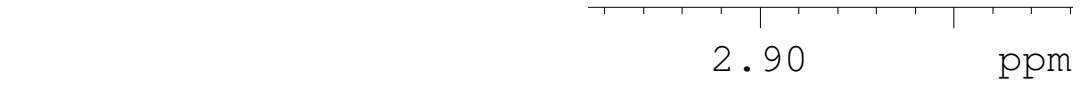

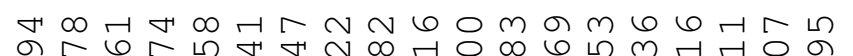
\% $\dot{\nabla} \dot{\gamma} \dot{\gamma} \dot{\gamma} \dot{\gamma} \dot{\sim} \dot{m} \dot{m} \dot{\sim} \dot{\sim} \dot{\sim} \dot{\sim} \dot{\sim} \dot{\sim} \dot{\sim} \sim$

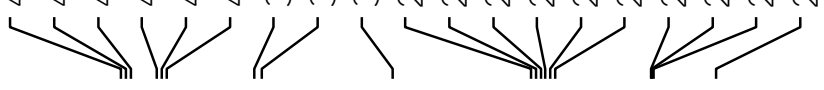

Proton NMR Spectrum (400 MHz, DMSO)

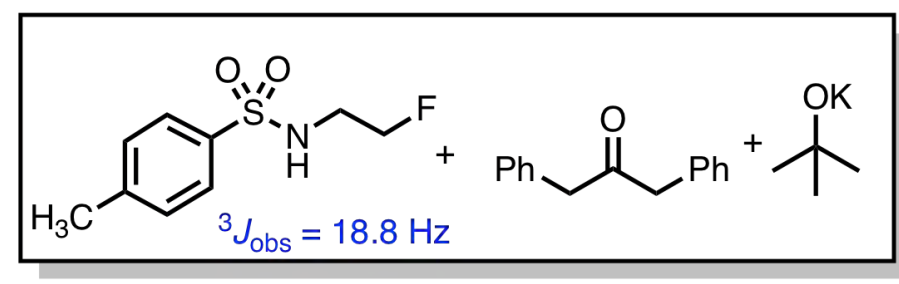

${ }^{3} J_{\mathrm{obs}}=18.8 \mathrm{~Hz}$

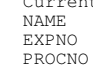

PROCN

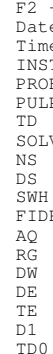
quisition Parameters
20190329
1232 $5 \mathrm{~mm}$ PABBO B

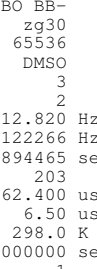

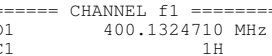
- Processing parameters $\begin{array}{lc}\text { SI } & 65536 \\ \text { SE } & 400.1300000 \mathrm{MHz} \\ \text { WDW } & \text { EM }\end{array}$

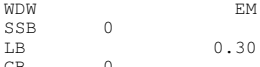

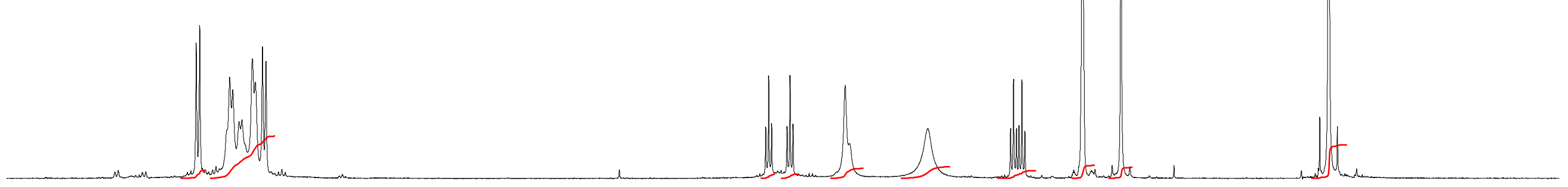

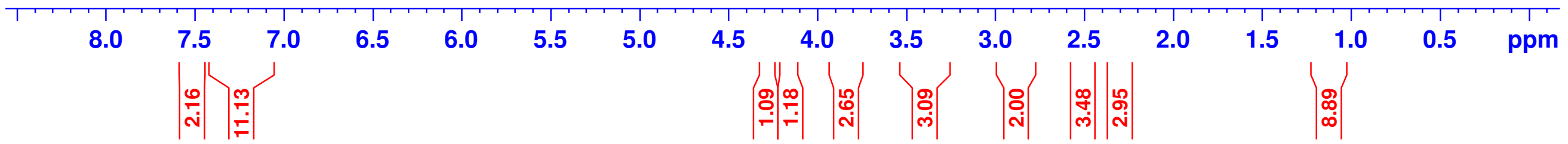




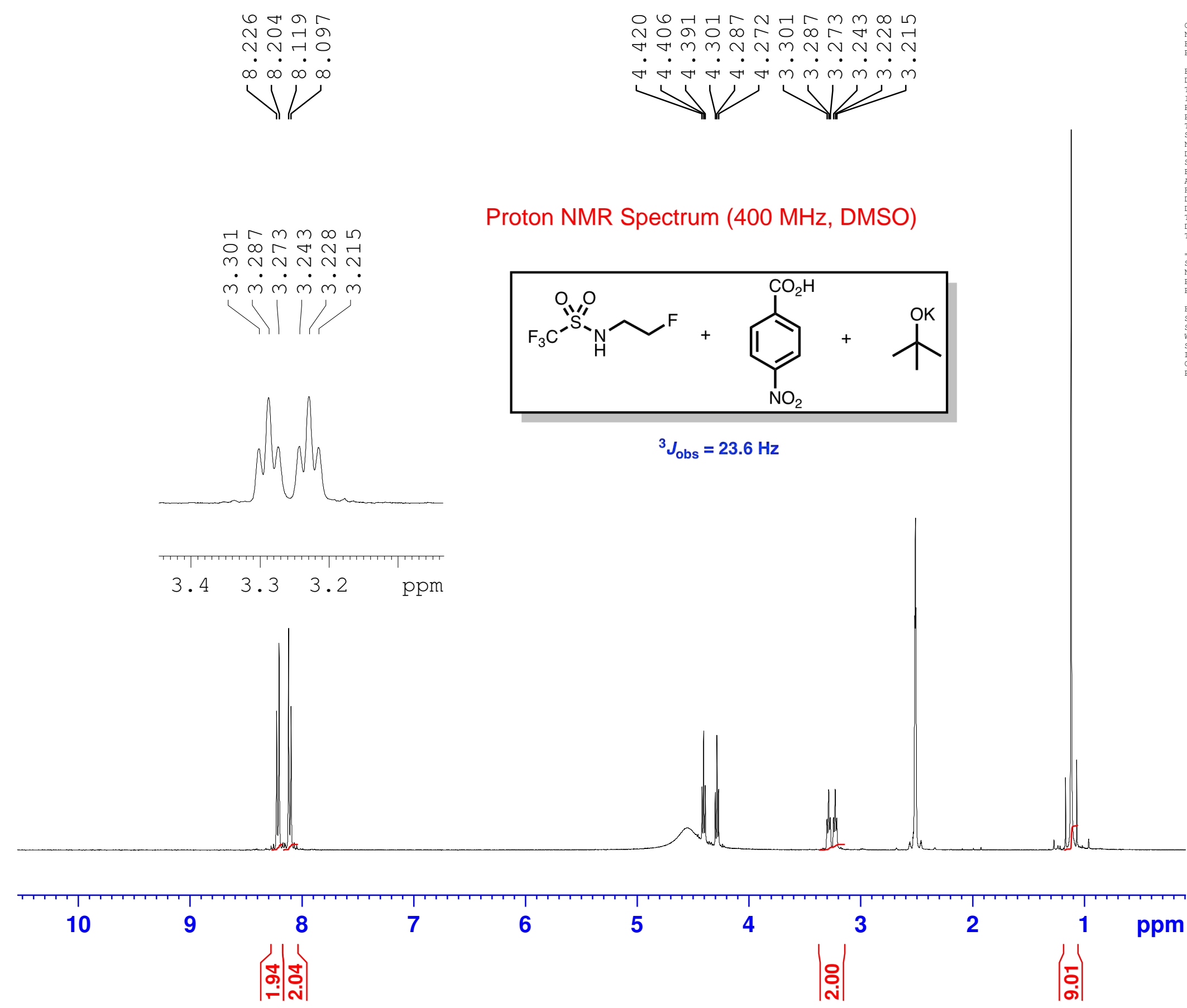




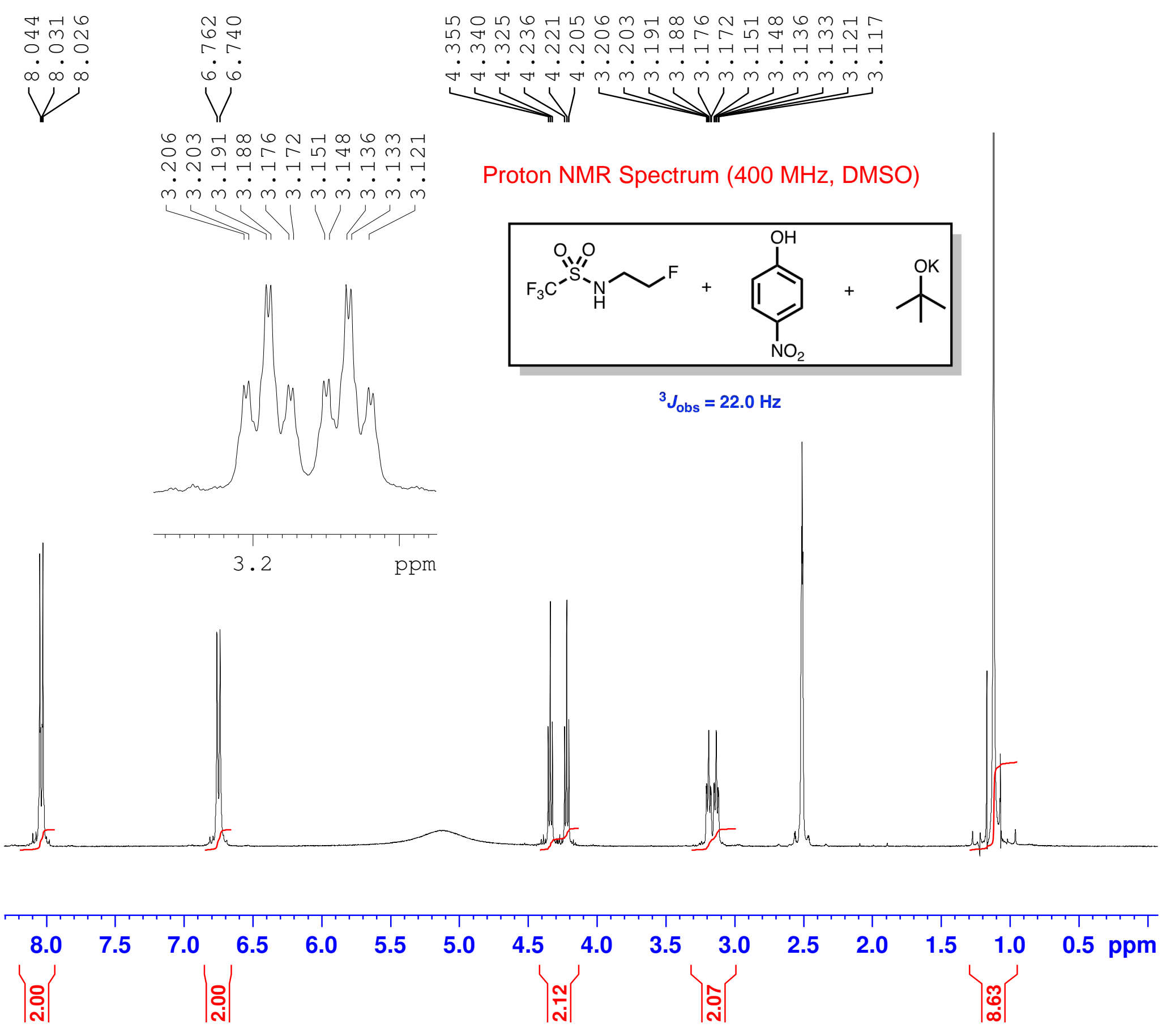

Current Data Parameters
NAME
F-CH2-CH2-NTf+4-nitrophenol2 EXPNO
PROCNO

F2 - Acquisition Parameters
Date_

INSTRUM

spect
$\mathrm{mm}$ PABBO BB-

TD

NS
DS

SWH

$\mathrm{AQ}$
$\mathrm{RG}$

$R G$
$D W$
$D E$
$T E$

TD 0

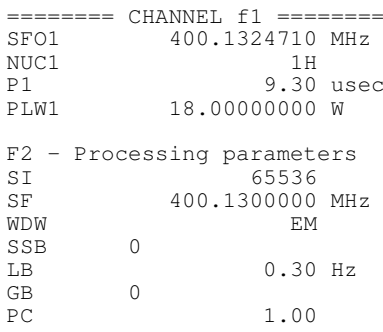

$0.122266 \mathrm{~Hz}$
$4.0894465 \mathrm{~Hz}$

62.400 usec

6.50 usec
$298.0 \mathrm{~K}$

$1.0000000 \mathrm{sec}$

00.1324710 MHz 
$\stackrel{m}{m} \infty \sim$

$m$ न

rer

VV

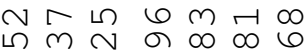

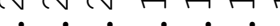

$\dot{m} \dot{m} \dot{m} \dot{m} \dot{m} \dot{m}$

11111

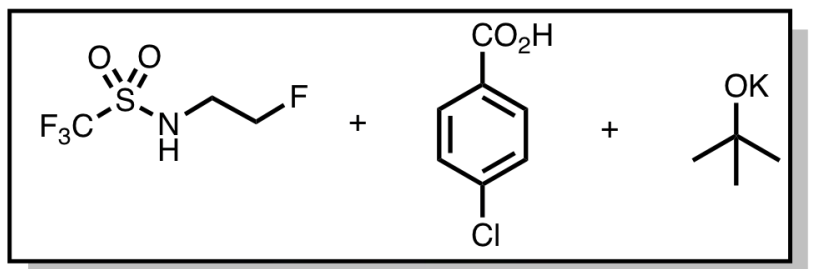

Proton NMR Spectrum (400 MHz, DMSO)

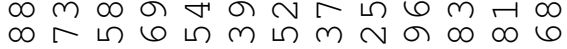

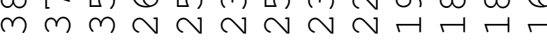

$\dot{\forall} \dot{\nabla} \dot{\nabla} \dot{\sigma} \dot{\sim} \dot{m} \dot{m} \dot{m} \dot{m} \dot{m} \dot{m}$

$\rightarrow 1$

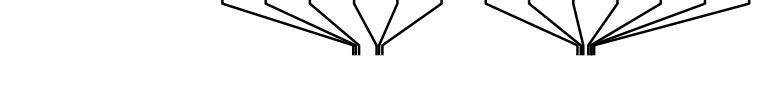

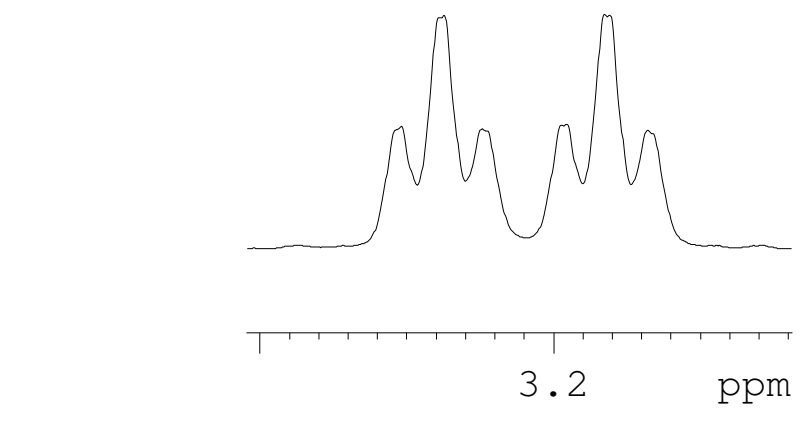

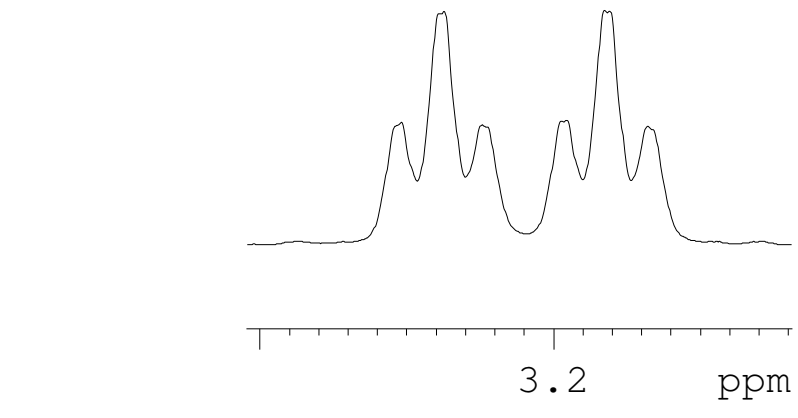

ppm

2 - Acquisition Parameters

$\begin{array}{lr}\text { Time } & 12.4 \\ \text { INSTRUM } & \text { spec } \\ \text { PROBHD } & 5 \mathrm{~mm} \text { PABBO BB- }\end{array}$

LVENT

DRES

8012.822
0.12226
0.72

084228

62.4200 usec
6950.0 usec
298.0

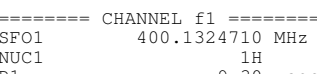

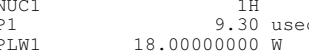

$\underset{\mathrm{SI}}{\mathrm{F}}-$ Processing parameters

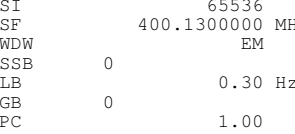

1.00

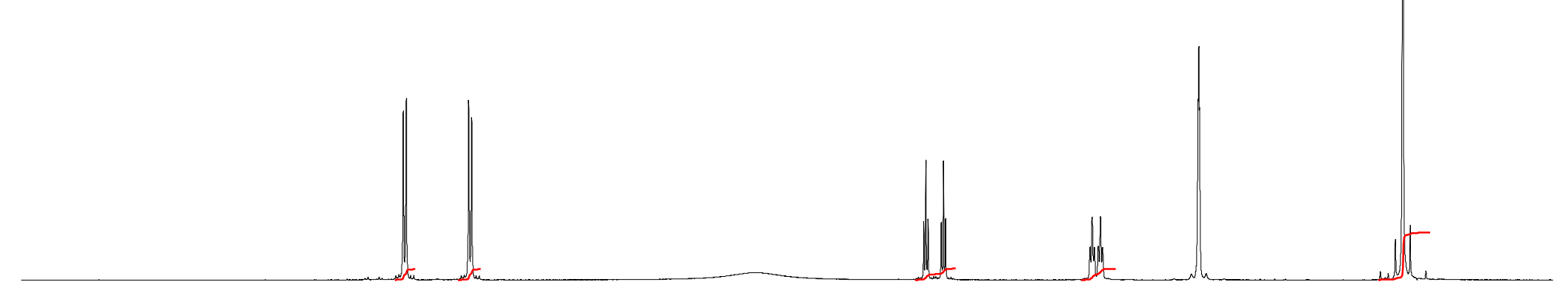

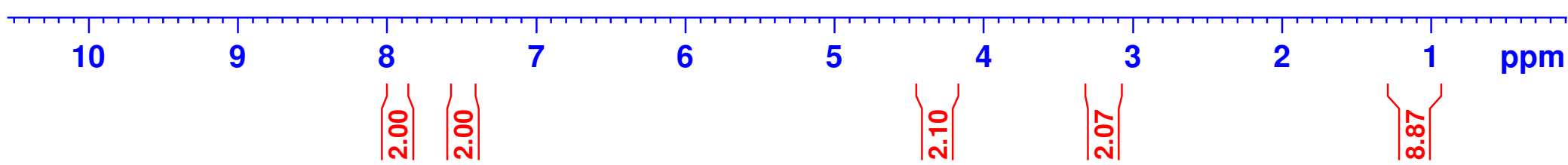




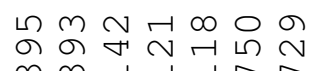

$m m \cdot$.

$\infty \infty \infty \infty \sim$

$1 /$

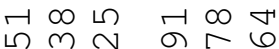

$m m m \sim \sim \sim$

$\dot{m} \dot{m} \dot{m} \dot{m} \dot{m}$

11111

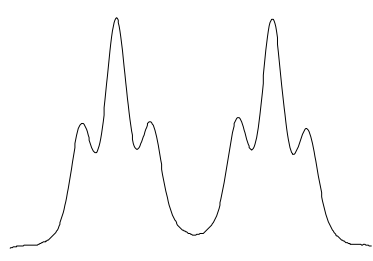

Proton NMR Spectrum (400 MHz, DMSO)

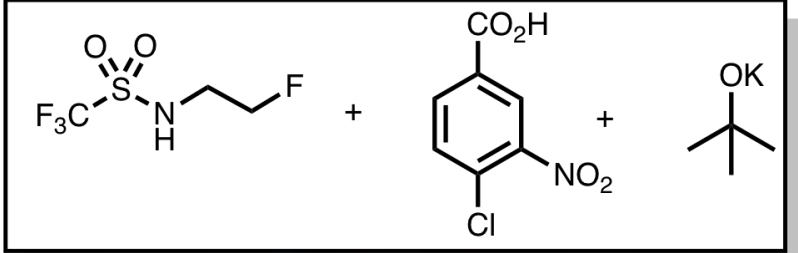

${ }^{3} J_{\mathrm{obs}}=24.0 \mathrm{~Hz}$ m の

$\forall \forall \forall m m m m m$

$\dot{\nabla \dot{r}} \dot{\gamma} \dot{\gamma} \dot{\gamma} \dot{\gamma} \dot{m} \dot{m} \dot{m} \dot{m} \dot{m}$

NII

3.3 ppm
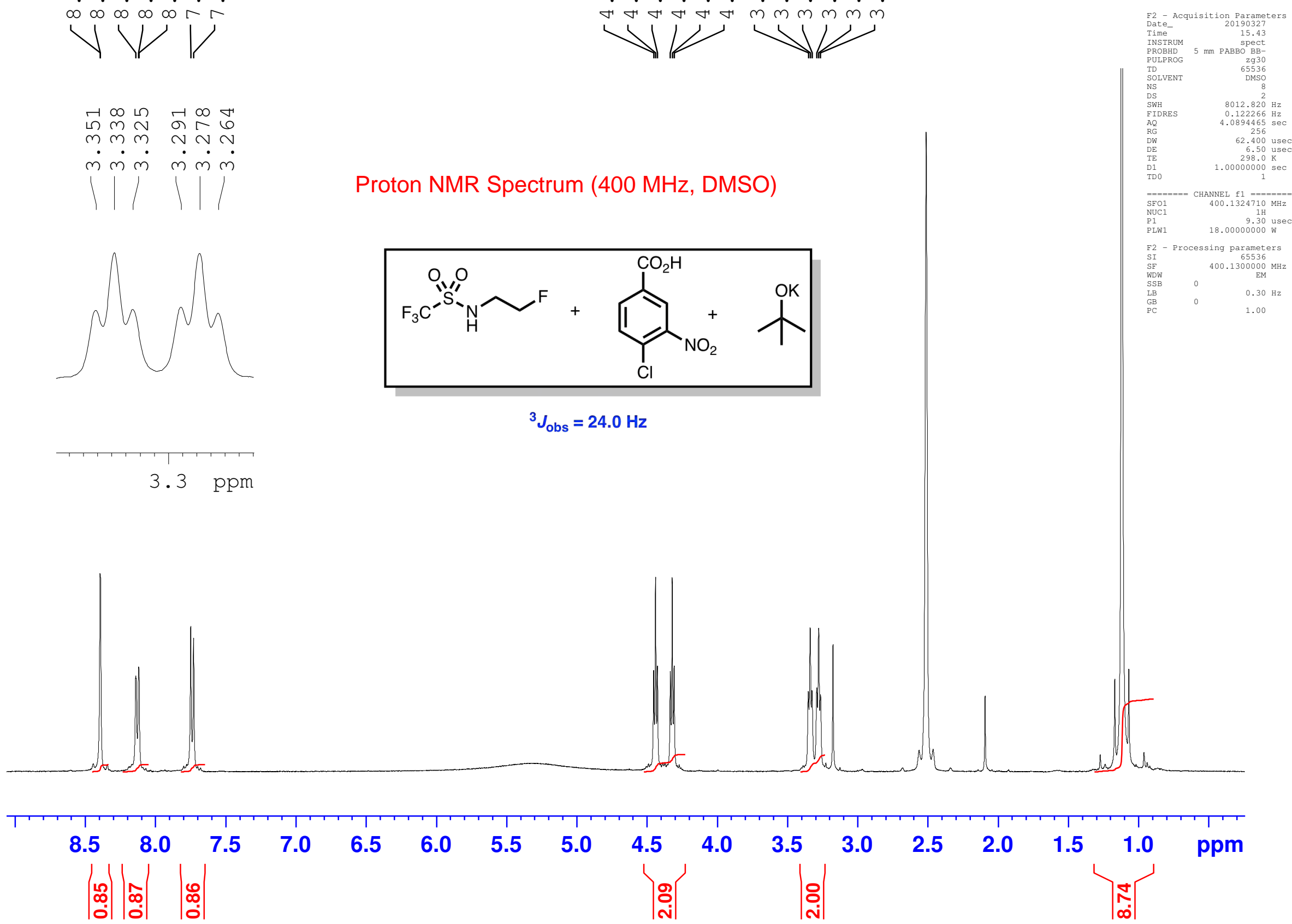


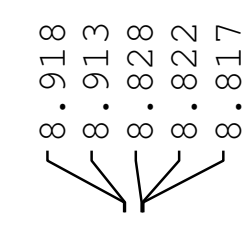

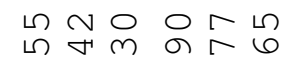

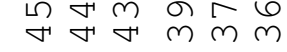
$\dot{m} \dot{m} \dot{m} \dot{m} \dot{m} \dot{m}$ 1111

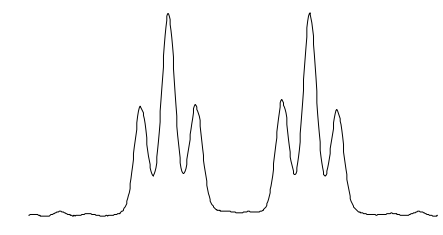

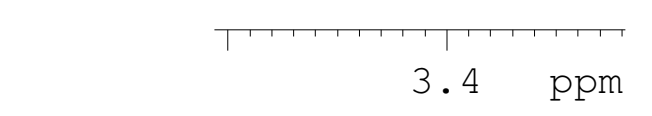

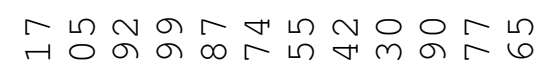
ㄷํำ $\dot{\forall} \dot{\forall} \dot{\gamma} \dot{\gamma} \dot{\gamma} \dot{m} \dot{m} \dot{m} \dot{m} \dot{m}$

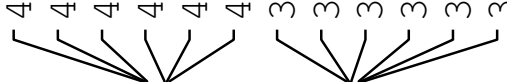

${ }^{3} \mathrm{~J}_{\mathrm{obs}}=26.0 \mathrm{~Hz}$

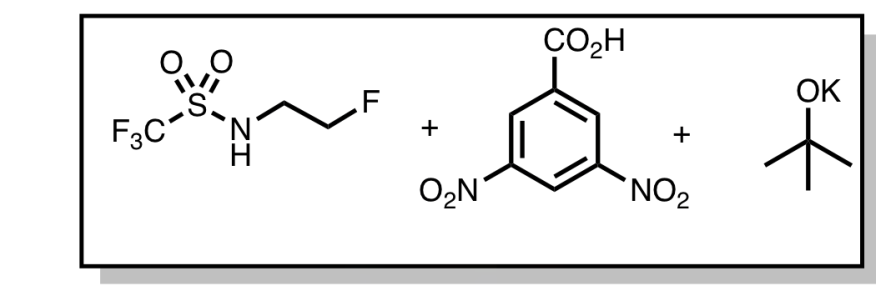

\section{Proton NMR Spectrum (400 MHz, DMSO)}

Current Data Parameters
NAME
F-CH2-CH2-NTf+3, 5-dintrobenzoic-acid

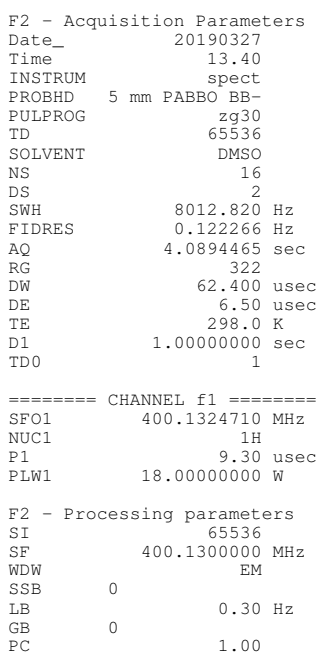

1.00
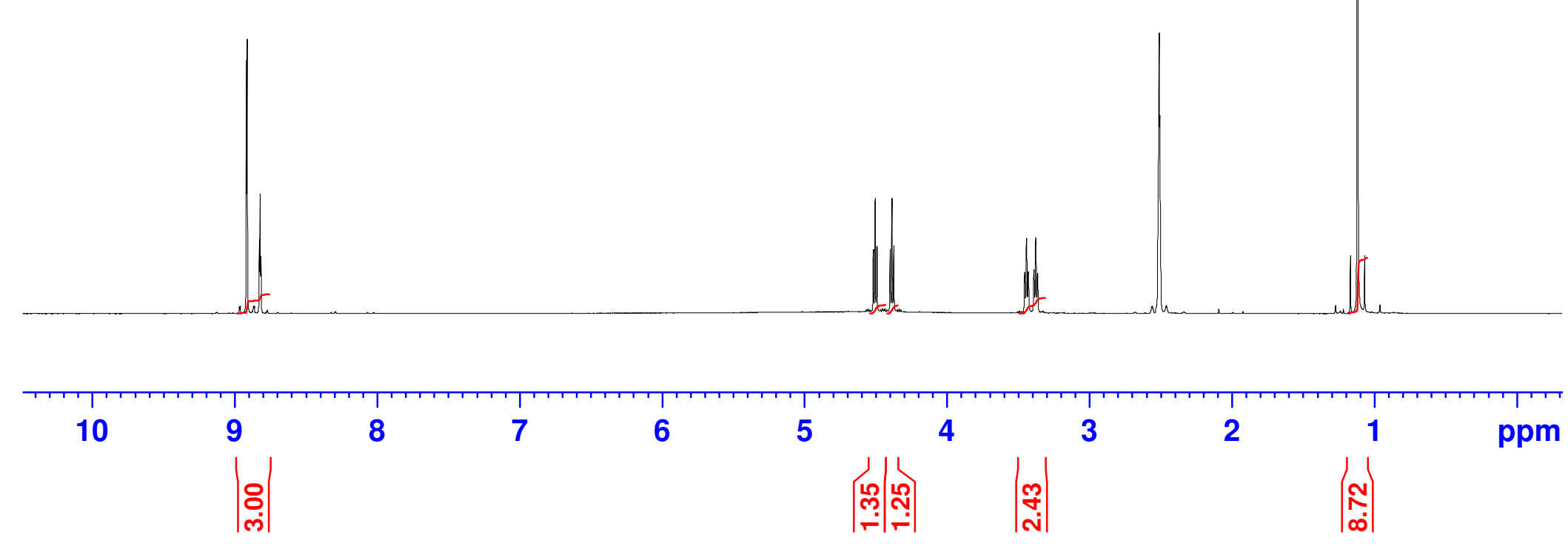


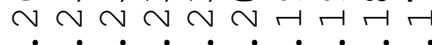

$\dot{m} \dot{m} \dot{m} \dot{m} \dot{m} \dot{m} \dot{m} \dot{m} \dot{m}$

$\longrightarrow 11$

\section{Proton NMR Spectrum (400 MHz, DMSO)}

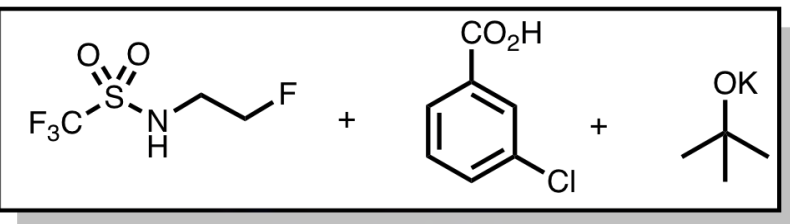

${ }^{3} J_{\mathrm{obs}}=22.8 \mathrm{~Hz}$
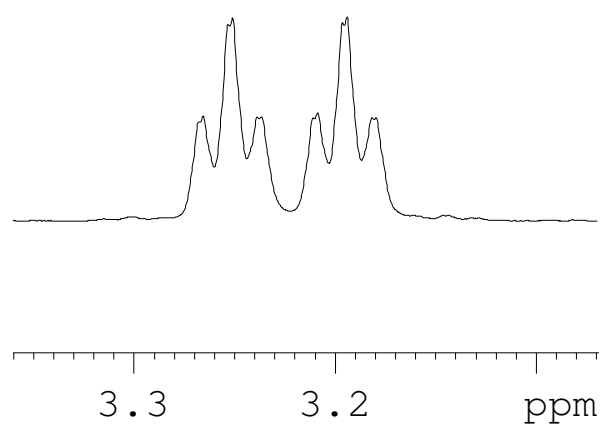

ppm
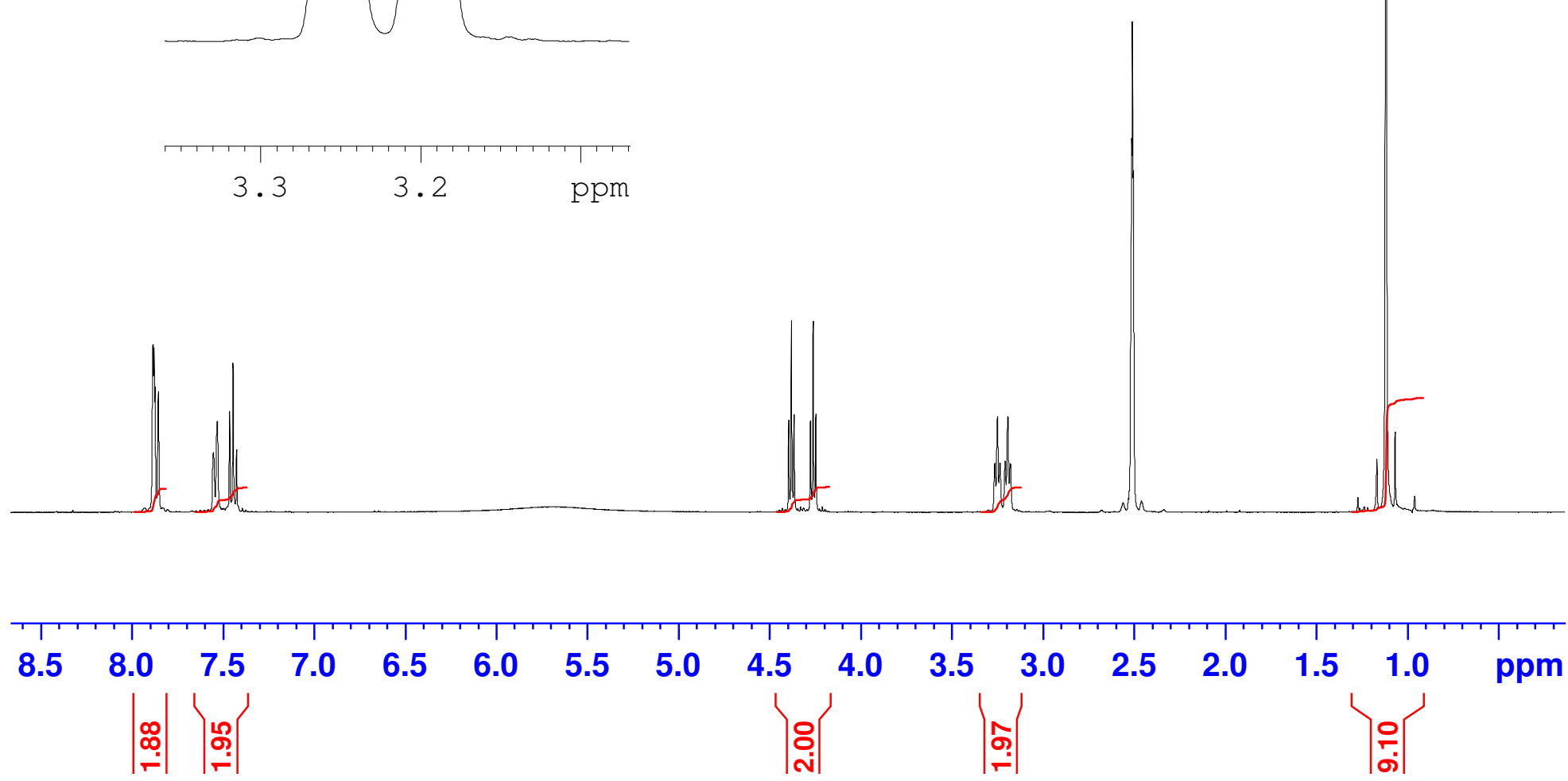